\title{
Congestion Games with Variable Demands
}

\author{
Tobias Harks* Max Klimm*广
}

\begin{abstract}
We initiate the study of congestion games with variable demands where the (variable) demand has to be assigned to exactly one subset of resources. The players' incentives to use higher demands are stimulated by non-decreasing and concave utility functions. The payoff for a player is defined as the difference between the utility of the demand and the associated cost on the used resources. Although this class of non-cooperative games captures many elements of real-world applications, it has not been studied in this generality, to our knowledge, in the past.

We study the fundamental problem of the existence of pure Nash equilibria (PNE for short) in congestion games with variable demands. We call a set of cost functions $C$ consistent if every congestion game with variable demands and cost functions in $C$ possesses a PNE. We say that $C$ is FIP consistent if every such game possesses the $\alpha$-Finite Improvement Property for every $\alpha>0$. Our main results are structural characterizations of consistency and FIP consistency for twice continuously differentiable cost functions. Specifically, we show

1. $C$ is consistent if and only if $C$ contains either only affine functions or only homogeneously exponential functions $\left(c(\ell)=a e^{\phi \ell}\right)$.

2. $C$ is FIP consistent if and only if $C$ contains only affine functions.

Our results provide a complete characterization of consistency of cost functions revealing structural differences to congestion games with fixed demands (weighted congestion games), where in the latter even inhomogeneously exponential functions are FIP consistent.

Finally, we study consistency and FIP consistency of cost functions in a slightly different class of games, where every player experiences the same cost on a resource (uniform cost model). We give a characterization of consistency and FIP consistency showing that only homogeneously exponential functions are consistent.
\end{abstract}

\section{Introduction}

Resource allocation problems play a key role in many applications. Whenever a set of resources needs to be matched to a set of demands, the goal is to find the most profitable or least costly allocation of the resources to the demands. Examples of such applications come from a wide range of areas, most prominently traffic networks [4, 28, 30, 32] and telecommunication networks [16, 17, 31]. In most of the above applications, the allocation of resources is determined by a finite number of independent players, each optimizing an individual objective function. A natural framework for analyzing such non-cooperative games are congestion

\footnotetext{
${ }^{*}$ Technische Universität Berlin, Institut für Mathematik, Straße des 17. Juni 136, 10623 Berlin, Germany. Email: \{harks, klimm\}@math.tu-berlin.de.

${ }^{\dagger}$ This research was supported by the Deutsche Forschungsgemeinschaft within the research training group 'Methods for Discrete Structures' (GRK 1408).
} 
games as introduced by Rosenthal [27]. In a congestion game, there is a set of resources and a pure strategy of a player consists of a subset of resources. The cost of a resource depends only on the number of players choosing the resource, and the private cost of a player is the sum of the costs of the chosen resources. Under these assumptions, Rosenthal proved the existence of a pure Nash equilibrium (PNE for short).

In the past, the existence of PNE has been analyzed in many variants of congestion games such as scheduling, routing, facility location, network design, each variant with unweighted and weighted players, see [1, 3, 5, 9, 14, 20]. Most of these previous works have a common feature: given a set of resources whose cost increases with increasing congestion, every player allocates a fixed demand to an available subset. While obviously important, such models do not take into account a fundamental property of many real-world applications: the intrinsic coupling between the quality or cost of the resources and the resulting demands for the resources. A prominent example of this coupling is the flow control problem in telecommunication networks. In this setting, players receive a non-negative utility from sending data and the perceived costs increase with congestion. In this and other examples, the demands will be reduced if the resources are congested, and increased if the resources are uncongested. Allowing for variable demand is, thus, a natural prerequisite for modeling the tradeoff between the benefit for demand, and the quality of the resource.

There is a large body of work addressing the issue of variable demands: [6, 19, 17, 29, 31] in the context of telecommunication networks and [4, 13] in the context of traffic networks. Most of these works assume that the (variable) demand may be fractionally distributed over the available subsets of resources. This assumption together with convexity assumptions on the cost and utility functions implies the existence of a PNE by Rosen's theorem [26]. Allowing a fractional distribution of the demand, however, is obviously not possible in many applications. For instance, the standard TCP/IP protocol suite uses single path routing, because splitting the demand comes with several practical complications, e.g., packets arriving out of order, packet jitter due to different paths delays etc.

We initiate the study of congestion games with variable demands, where the (variable) demand has to be assigned to exactly one subset of resources. We impose standard economic assumptions (cf. [13, 17, 29]) that every player is associated with a non-decreasing and concave utility function measuring the utility for the demand. The payoff for a player is defined as the difference between the utility and the associated congestion cost on the used resources where the cost of a player on a resource is the product of the cost function, and the player's demand.

There are two fundamental goals from a system design perspective: (i) the system must be stabilizable, that is, there must be a stable point (PNE) from which no player wants to unilaterally deviate; (ii) myopic play of the players should guide the system to a stable state. Because the utility functions are only known to the players (as they are private information) and not available to the system designer, we study the above two issues with respect to the used cost functions (which represent the technology associated with the resources, e.g., queuing discipline at routers, latency function in transportation networks, etc.). Let $C$ be a set of cost functions and let $\mathcal{G}(C)$ be the set of all congestion games with variable demands and cost functions in $C$. We say that $C$ is consistent if every game in $\mathcal{G}(C)$ possesses a PNE. We say that $C$ is FIP consistent if for every game in $\mathcal{G}(C)$, every sequence of unilateral $\alpha$-improvements (improvements that add at least $\alpha$ to the payoff of the deviating player, see [21]) is finite. The main goal of this work is to investigate consistency and the $\alpha$-FIP of the used cost functions.

Connection to Prior Work. Already earlier work recognized the importance of understanding the impact of the cost structure on the existence of PNE and the $\alpha$-FIP in variants of congestion games, see [2, 8, 18, 23, 26]. The existence of PNE with respect to the cost structure also plays a fundamental role in the vast literature on Cournot oligopoly games in the economic theory of imperfect competition. In a Cournot oligopoly game, there is a set of firms each producing quantities so as to satisfy an elastic demand. The production cost for every player is modeled by a convex cost function and the interaction between the firms 
comes from the price determination mechanism which is dependent on the total supply on the market. Note that congestion games with variable demands can be interpreted as a natural generalization of the Cournot oligopoly game. In [15] it is proved that Cournot oligopoly games are basically equivalent (in terms of the set of Nash equilibria) to congestion games with variable demand and a single resource (which are termed Cournot oligopsonies in [15]). The model proposed in this article is more general since the strategy space of players involves not only quantities but also sets of allowable subsets of resources (markets).

The pioneering work of Cournot [7] established the existence of an equilibrium point for this model assuming zero costs and concave inverse demand functions. Further existence results for more general cost functions have been established by many researchers, among others [22, 25].

In the seminal paper by Orda et al. [23], the authors address the issue of uniqueness of PNE in congestion games with splittable demands. They give sufficient conditions for uniqueness of PNE for several classes of cost functions. In the final section of their paper, the following question is raised (we quote from the paper):

"Several other open questions of practical value deserve attention. For example, in many networks users are restricted to route their flow along a single path with strict rules of changing them. Under such circumstances an NEP may not exist at all and complicated oscillatory behavior is likely to arise."

Our Results. We initiate the study of congestion games with variable demands and focus on the existence of pure Nash equilibria and the finite improvement property. Our main results are structural characterizations of the existence of PNE and the $\alpha$-FIP with respect to the cost structure. Specifically, we show the following:

1. Let $C$ be a set of non-negative, strictly increasing and twice differentiable cost functions. We prove that $C$ is consistent if and only if exactly one of the following cases hold: (a) $C$ contains only affine functions $c(\ell)=a_{c} \ell+b_{c}$ with $a_{c}>0, b_{c} \geq 0 ;(b) C$ contains only homogeneously exponential functions such that $c(\ell)=a_{c} e^{\phi \ell}$ for some $a_{c}, \phi>0$, where $a_{c}$ may depend on $c$, while $\phi$ must be equal for all $c \in C$. Moreover, we characterize the $\alpha$-FIP in congestion games with variable demands. We prove that $C$ is FIP consistent if and only if $C$ contains only affine functions. The formal results appear as Theorems 4.4 and 4.5 .

We show that our results remain valid for games with network structure and, thus, our characterizations settle the open questions raised by Orda et al. [23].

2. We then investigate a slightly different class of games that we term uniform games. They differ from the previously studied games in the definition of the players' payoff functions. In uniform games the cost for a player on a resource is not multiplied with the demand of that player. For fixed demands, uniform games have been studied by many researchers, e.g., [8, 9, 14, 20]. Considering uniform cost structures is motivated by a series of real-world applications. In large-scale telecommunication networks, it is highly desirable to charge every player the same cost regardless of the actual resource consumption of every player, because every resource needs only to communicate a single value to the players giving rise to an efficient and scalable implementation [31]. In scheduling applications, the cost function is frequently used to model the achieved makespan which is (under round-robin processing) equal for every job on the same resource.

Our second main result provides a complete characterization of consistency of a set of cost functions for the uniform cost model. We prove that $C$ is consistent w.r.t. uniform cost games if and only if $C$ contains homogeneously exponential functions such that $c(\ell)=a_{c} e^{\phi \ell}$ for some $a_{c}, \phi>0$, where $a_{c}$ may depend on $c$, while $\phi$ must be equal for all $c \in C$. Surprisingly, this characterization reveals that uniform games need not possess a PNE, even if costs are affine. We also characterize the $\alpha$-FIP in the uniform cost model. We prove that $C$ is FIP consistent if and only if $C=\emptyset$. For the case of homogeneously exponential cost functions, however, we derive an improvement dynamic converging to a PNE, thus, showing that the improvement graphs of the resulting games are weakly acyclic. 
Table 1: Existence of PNE and the $\alpha$-FIP in congestion games with variable demands with proportional costs and uniform costs. Note the fundamental structural difference to weighted congestion games (with fixed demands) where in the proportional and uniform cost model there is always a PNE and the $\alpha$-FIP for affine and inhom. exponential cost functions, see [8, 11, 12, 24].

\begin{tabular}{lcccccc}
\hline & \multicolumn{2}{c}{ variable demands } & \multicolumn{2}{c}{ variable demands } & \multicolumn{2}{c}{ fixed demands } \\
& \multicolumn{2}{c}{ proportional costs } & \multicolumn{2}{c}{ uniform costs } & \multicolumn{2}{c}{ prop. \& unif. costs } \\
cost functions & PNE & $\alpha$-FIP & PNE & $\alpha$-FIP & PNE & $\alpha$-FIP \\
\hline affine & yes & yes & no & no & yes [8] & yes [8] \\
hom. exp. & yes & no & yes & no & yes [24] & yes [24] \\
\hline inhom. exp. & no & no & no & no & yes [12] & yes [12] \\
non aff. \& non exp. & no & no & no & no & no [11] & no [11] \\
\hline
\end{tabular}

Our results are summarized in Table 1, All proofs missing in this extended abstract as well as some examples are presented in the Appendix.

Main Ideas and Outline. After introducing the basic model in Section 2, we prove the "only if" direction of our first result (Theorem 4.4) in Section 3. In the proof, we first establish a connection between weighted congestion games and congestion game with variable demands. Given a weighted congestion without PNE, we derive a congestion game with variable demands using the same cost functions that also has no PNE. The proof idea relies on a careful design of feasible (concave and differentiable) utility functions which preserve the improvement cycles of the original weighted congestion game. Thus, we can use an earlier result of [11] stating that a set of cost functions is consistent for weighted congestion games if and only if this set contains either affine functions or certain exponential functions. The hard part of completing the "only if" direction lies in excluding inhomogeneously exponential cost functions. We prove that these functions are not consistent by studying a class of congestion games with fixed resource dependent demands. We identify a subclass of these games with inhomogeneously exponential cost functions for which we construct a congestion game with variable demands. We show that the thus constructed game fulfills the invariant of preserved improvement cycles with respect to the original game. While this part of the proof is perhaps the most technical, we obtain as a side-product of our analysis a novel characterization of the existence of PNE for congestion games with resource dependent demands showing that only affine functions are consistent.

In Section 4, the "if" part is proven. We introduce a novel potential function concept that we term essential generalized ordinal potentials. The idea is to require that there is a real-valued function that must increase only for a subset of improving moves. We further introduce local essential potentials, where this property must hold only for a global maximum of the potential. For games with homogeneously exponential cost functions, we derive a local essential potential completing the "if" direction (games with affine costs are exact potential games). In Section 5, we investigate a class of games that we term uniform congestion games with variable demands. We give similar characterizations of consistency of cost functions, yielding that only homogeneously exponential cost functions are consistent. We prove the "if" direction by deriving an essential potential. We conclude the paper in Section 6 by presenting new research directions.

\section{Preliminaries}

Congestion games with variable demands are strategic games $G=\left(N, \bar{X},\left(\pi_{i}\right)_{i \in N}\right)$, where $N=\{1, \ldots, n\}$ is the non-empty and finite set of players, $\bar{X}=\chi_{i \in N} \bar{X}_{i}$ is the non-empty set of states or strategy profiles, and $\pi_{i}: \bar{X} \rightarrow \mathbb{N}$ is the individual payoff function that specifies the payoff value of player $i$ for each state $\bar{X} \in \bar{X}$. We define strategies and payoff functions using the general notion of a congestion model. A tuple 
$\mathcal{M}=\left(N, R, X,\left(c_{r}\right)_{r \in R}\right)$ is called a congestion model if $N$ is a set of players, $R$ is a finite set of resources, and $X=X_{i \in N} X_{i}$ is the set of configurations. For each player $i \in N$, the set $X_{i} \subseteq 2^{R}$ is a finite collection of subsets of $R$. Every resource $r \in R$ is endowed with a cost function $c_{r}: \mathbb{R}_{\geq 0} \rightarrow \mathbb{R}_{\geq 0}$. In a congestion game with variable demands, every player $i \in N$ is allowed to choose a configuration $x_{i} \in X_{i}$ and a non-negative demand $d_{i} \in \mathbb{R}_{\geq 0}$ that she places on all resources in $x_{i}$. The incentive to use higher demands is stimulated by a utility function $U_{i}: \mathbb{R}_{\geq 0} \rightarrow \mathbb{R}_{\geq 0}$ that measures the benefit that player $i$ receives from choosing a certain demand. Note that this benefit is independent of the resources chosen and depends solely on the chosen demand.

Let $\mathcal{M}=\left(N, R, X,\left(c_{r}\right)_{r \in R}\right)$ be a congestion model and let $\left(U_{i}\right)_{i \in N}$ be a collection of utility functions. We define a congestion game with variable demands and proportional costs as the game $G(\mathcal{M})=(N, \bar{X}, \pi)$, where $\bar{X}=\left(X, \mathbb{R}_{\geq 0}\right), \pi=\left(\pi_{i}\right)_{i \in N}$ and $\pi_{i}(x, d)=U_{i}\left(d_{i}\right)-\sum_{r \in x_{i}} d_{i} c_{r}\left(\ell_{r}(x, d)\right)$, and $\ell_{r}((x, d))=\sum_{j \in N: r \in x_{j}} d_{j}$. We call $\ell_{r}(x, d)$ the load on resource $r$ under strategy $(x, d)$. Note that these games are maximization games. A configuration $x \in X$ together with a demand profile $d \in \mathbb{R}_{\geq 0}^{n}$ forms a strategy profile $\bar{x}=(x, d)$. Note that proportional costs have been used before in [10] for the case of weighted congestion games.

We use standard game theory notation; for a set $S \subseteq N$ we denote by $-S$ its complement and by $\bar{X}_{S}=\chi_{i \in S} \bar{X}_{i}$ we denote the set of strategy profiles of players in $S$. Instead of $\bar{X}_{-\{i\}}$ we will write $\bar{X}_{-i}$, and with a slight abuse of notation we will sometimes write a strategy profile as $\bar{x}=\left(\bar{x}_{i}, \bar{x}_{-i}\right)$ meaning that $\bar{x}_{i} \in \bar{X}_{i}$ and $\bar{x}_{-i} \in \bar{X}_{-i}$.

For a constant $\alpha \geq 0$, a pair $\left(\bar{x},\left(\bar{y}_{i}, \bar{x}_{-i}\right)\right) \in \bar{X} \times \bar{X}$ is called an $\alpha$-improving move of player $i$ if $\pi_{i}(\bar{x})+\alpha<$ $\pi_{i}\left(\bar{y}_{i}, \bar{x}_{-i}\right)$. We denote by $I^{\alpha}(i)$ the set of $\alpha$-improving moves of player $i \in N$, and we set $I^{\alpha}=\bigcup_{i \in N} I^{\alpha}(i)$. We call a sequence of strategy profiles $\gamma=\left(x^{0}, x^{1}, \ldots\right)$ an $\alpha$-improvement path if every tuple $\left(x^{k}, x^{k+1}\right) \in I^{\alpha}$. A strategy profile $\bar{x} \in \bar{X}$ is a pure Nash equilibrium, or PNE for short, if $(\bar{x}, \bar{y}) \notin I^{0}$ for all $\bar{y} \in \bar{X}$. $G$ has the $\alpha$-finite improvement property ( $\alpha$-FIP) if every $\alpha$-improvement path is finite. Let $C$ be a class of cost functions. We call $C$ consistent w.r.t. congestion games with variable demands (or simply consistent) if every congestion game with variable demands and cost functions in $C$ admits a PNE. $C$ is FIP consistent if every congestion game with variable demands and cost functions in $C$ has the $\alpha$-FIP.

The following two assumptions contain mild restrictions on feasible utility functions and cost functions and are standard in the literature, see [13, 17, 29].

Assumption 2.1. For every resource $r \in R$ the cost function $c_{r}: \mathbb{R}_{\geq 0} \rightarrow \mathbb{R}_{\geq 0}$ is twice continuously differentiable and strictly increasing.

We denote by $C^{2}\left(\mathbb{R}_{\geq 0}\right)$ the set of functions satisfying Assumption 2.1.

Assumption 2.2. For every player $i \in N$ the utility function $U_{i}: \mathbb{R}_{\geq 0} \rightarrow \mathbb{R}_{\geq 0}$ is differentiable, nondecreasing and concave.

Remark 2.3. In contrast to most of the works in the area of Cournot games or congestion games with splittable demands (e.g.,[13, 17, 23]), we do not assume semi-convexity of cost functions.

\section{Necessary Conditions}

At first we present some simple but useful observations. It is easy to see that consistent cost functions cannot have bounded marginal costs. Formally, if $C$ is consistent, then $c(x)+x c^{\prime}(x) \rightarrow \infty$ as $x \rightarrow \infty$ for all $c \in C$. To see this, assume that there is $c \in C$ with $c(x)+x c^{\prime}(x)<M$ for some $M \in \mathbb{R}_{>0}$. Consider a game with one resource and cost function $c$ and one player with utility function $U(x)=(M+1) x$. The payoff of the player increases with the demand, thus, this game does not admit a PNE.

Moreover, the following lemma will be useful throughout this paper. It simply uses the first-order optimality condition of a PNE. 
Lemma 3.1. In any $P N E\left(x^{*}, d^{*}\right)$ of a congestion game with variable demands $U_{i}^{\prime}\left(d_{i}^{*}\right)=\frac{\partial d_{i}^{*} \sum_{r \in x_{i}} c_{r}\left(\ell_{r}\left(x^{*}, d^{*}\right)\right)}{\partial d_{i}^{*}}$ for all $i \in N$ with $d_{i}^{*}>0$, and $U_{i}^{\prime}\left(d_{i}^{*}\right) \leq \frac{\partial d_{i}^{*} \sum_{r \in x_{i}} c_{r}\left(\ell_{r}\left(x^{*}, d^{*}\right)\right)}{\partial d_{i}^{*}}$ for all $i \in N$ with $d_{i}^{*}=0$.

Necessity of Affine or Exponential Costs. First, we establish a connection between congestion games with variable demands and weighted congestion games. We will exploit this connection to show that consistent cost functions for congestion games with variable demands must be either affine or exponential.

Let $\mathcal{M}=\left(N, R, X,\left(c_{r}\right)_{r \in R}\right)$ be a congestion model and $\left(d_{i}^{\mathrm{w}}\right)_{i \in N}$ be a vector of demands with $d_{i}^{\mathrm{w}} \in \mathbb{R}_{>0}$. The corresponding weighted congestion game is the strategic game $G^{\mathrm{w}}(\mathcal{M})=(N, X, \pi)$, where $\pi$ is defined as $\pi=\chi_{i \in N} \pi_{i}, \pi_{i}(x)=\sum_{r \in x_{i}} d_{i}^{\mathrm{w}} c_{r}\left(\ell_{r}(x)\right)$ and $\ell_{r}(x)=\sum_{j \in N: r \in x_{j}} d_{j}^{\mathrm{w}}$. Note that these games are cost minimization games. We will show that whenever there is a weighted congestion game $G^{\mathrm{w}}(\mathcal{M})$ that does not admit a PNE, then there is also a congestion game with variable demands $G(\mathcal{M})$ under the same congestion model without a PNE. The proof of this result is constructive, i.e., given the weighted congestion game $G^{\mathrm{w}}(\mathcal{M})$ and the corresponding vector of demands $d_{i}^{\mathrm{w}}$, we construct a congestion game with variable demands not possessing a PNE.

Note that the main difficulty lies in the fact that in congestion games with variable demands the strategy space is strictly larger than in weighted congestion games. We overcome this issue by designing for every player $i$ a utility function $U_{i}$ that allows us to restrict a priori the set of equilibrium demands of player $i$ to a small environment $\left(t_{i}-\sigma, t_{i}+\sigma\right)$ around a target demand $t_{i}$. To this end, recall that Lemma 3.1 establishes that in every PNE player $i$ 's marginal utility equals her marginal cost given that her demand is strictly positive. Our basic idea is to define the utility function $U_{i}$ of player $i$ such that the following two properties are guaranteed: (i) player $i$ always has an interest to play a positive demand; (ii) for every possible value $C^{\prime}$ of player $i$ 's marginal costs, the equation $U_{i}^{\prime}\left(d_{i}\right)=C^{\prime}$ is met only for $d_{i} \in\left(t_{i}-\sigma, t_{i}+\sigma\right)$. As we will see, we can find smoothed 2 -wise linear utility functions that suit our purposes.

We define the piecewise linear function through the sequence of points $\left(\left(\tau_{0}, v_{0}\right),\left(\tau_{1}, v_{1}\right), \ldots,\left(\tau_{k}, v_{k}\right)\right)$ as the function

$$
g\left(\left(\tau_{0}, v_{0}\right), \ldots,\left(\tau_{k}, v_{k}\right)\right)(x)= \begin{cases}v_{i}+\frac{v_{i}-v_{i-1}}{\tau_{i}-\tau_{i-1}}\left(x-\tau_{i}\right), & \text { if } x \in\left[\tau_{i}, \tau_{i+1}\right) \text { for some } i \in\{0, k-1\}, \\ v_{k}, & \text { if } x \in\left[\tau_{k},+\infty\right) .\end{cases}
$$

Since we are interested in obtaining non-decreasing and concave functions we will assume in the following that the sequence of slopes $\left(\frac{v_{i}-v_{i-1}}{\tau_{i}-\tau_{i-1}}\right)_{i=1, \ldots, k}$ is positive and non-increasing. To obtain differentiable functions, we choose a sufficiently small $\sigma>0$ and replace the function $g$ on every set $\left(\tau_{i}-\sigma, \tau_{i}+\sigma\right), i=1, \ldots, k$ by a non-decreasing and differentiable function such that the functional values and first derivatives in $\tau_{i}-\sigma$ and $\tau_{i}+\sigma$ comply. For instance, we can choose a suitable quadratic function on every interval $\left(\tau_{i}-\sigma, \tau_{i}+\sigma\right)$, see the following example.

Example 1. Let $g_{\left(\left(\tau_{1}, v_{1}\right), \ldots,\left(\tau_{k}, v_{k}\right)\right)}$ be a non-decreasing and concave piecewise linear function and let $0<\sigma<$ $\left(\min _{i=1, \ldots, k} \tau_{i}-\tau_{i-1}\right) / 2$ be a smoothening parameter. We regard the function $g_{\left(\left(\tau_{1}, v_{1}\right), \ldots,\left(\tau_{k}, v_{k}\right)\right)}^{\sigma}: \mathbb{R}_{\geq 0} \rightarrow \mathbb{R}_{\geq 0}$ defined as

$$
g_{\left(\left(\tau_{1}, v_{1}\right), \ldots,\left(\tau_{k}, v_{k}\right)\right)}^{\sigma}(x)= \begin{cases}\frac{1}{4 \sigma}\left(s_{i}-s_{i-1}\right)\left(x-\tau_{i}+\sigma \frac{s_{i}+s_{i-1}}{s_{i}-s_{i-1}}\right)^{2}+v_{i}+\sigma s_{i}-\frac{\sigma s_{i}^{2}}{s_{i}-s_{i-1}}, & \text { if } x \in\left(\tau_{i}-\sigma, \tau_{i}+\sigma\right), i=1, \ldots, k \\ g_{\left(\left(\tau_{1}, v_{1}\right), \ldots,\left(\tau_{k}, v_{k}\right)\right)}(x), & \text { else. }\end{cases}
$$

Remark that the quadratic functions on $\left(\tau_{i}-\sigma, \tau_{i}+\sigma\right), i=1, \ldots, k$ are chosen such that the smoothed piecewise linear function $g_{\left(\left(\tau_{1}, v_{1}\right), \ldots,\left(\tau_{k}, v_{k}\right)\right)}^{\sigma}$ is continuously differentiable. Two such functions are shown in Figure 1

We denote the corresponding smoothed piecewise linear function by $g^{\sigma}\left(\left(\tau_{0}, v_{0}\right), \ldots,\left(\tau_{k}, v_{k}\right)\right)$. By designing special smoothed 2-wise linear functions, we obtain the following lemma. 


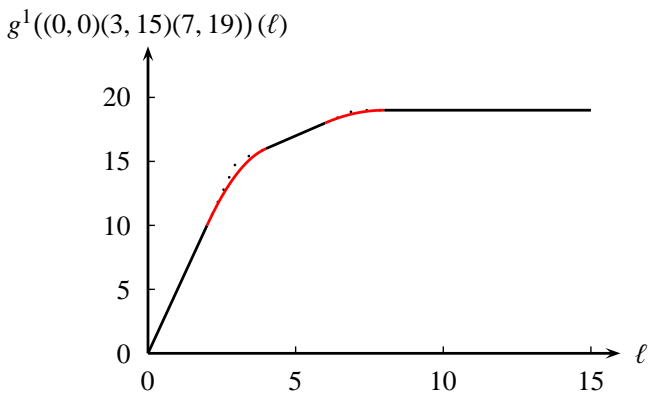

(a) Smoothed 3-wise linear function

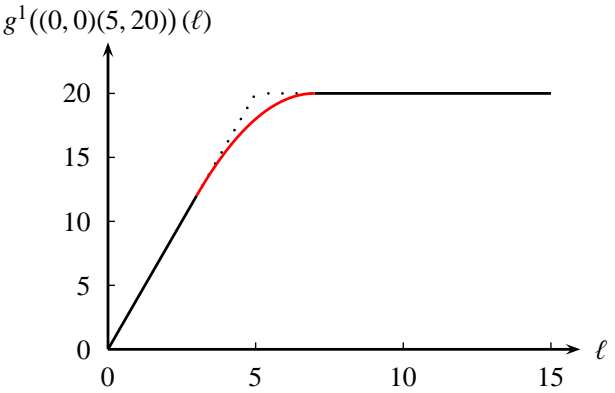

(b) Smoothed 2-wise linear function

Figure 1: Plot of the smoothed 3-wise linear function $\left.g^{1}((0,0)(3,15)(7,19))\right)$ and of the smoothed 2-wise linear function $g^{1}((0,0)(5,20))$.

Lemma 3.2. Let $\mathcal{M}$ be a congestion model, $\left(t_{i}\right)_{i \in N} \in \mathbb{R}_{>0}^{n}$ a vector of target demands with $t_{\min }=\min _{i \in N}\left\{t_{i}\right\}$ and $t_{\max }=\max _{i \in N}\left\{t_{i}\right\}$. Moreover, let $M>\max \left\{\frac{2 \sum_{r \in R} c_{r}(T)}{t_{\min }}, \sum_{r \in R}\left(c_{r}(T)+\left(t_{\max }+\sigma\right) \max _{\ell \in\left[t_{\min }, T\right]} c_{r}^{\prime}(\ell)\right)\right\}$, where $T=\sum_{j \in N}\left(t_{j}+\sigma\right)$ and $\sigma<t_{\min } / 2$. Let $G$ be the congestion game with variable demands and utilities $U_{i}=g_{\left((0,0),\left(t_{i}, M t_{i}\right)\right)}^{\sigma}$ for all $i \in N$. Then, $d_{i}^{*} \in\left(t_{i}-\sigma, t_{i}+\sigma\right)$ for all $i \in N$ and every PNE $\left(x^{*}, d^{*}\right)$ of $G$.

Proof. For contradiction, suppose there is a PNE $\bar{x}^{*}=\left(x^{*}, d^{*}\right)$ of $G$ and $i \in N$ with $d_{i}^{*} \notin\left(t_{i}-\sigma, t_{i}+\sigma\right)$. First, assume $d_{i}^{*}>t_{i}+\sigma$. As $c_{r}$ is strictly increasing, we may assume without loss of generality that there is $r \in x_{i}$ with $c_{r}\left(d_{i}^{*}\right)>0$. Lemma 3.1 implies $0=U_{i}^{\prime}\left(d_{i}^{*}\right)=\sum_{r \in x_{i}^{*}}\left(c_{r}\left(\ell_{r}\left(\bar{x}^{*}\right)\right)+d_{i}^{*} c_{r}^{\prime}\left(\ell_{r}\left(\bar{x}^{*}\right)\right)>0\right.$, which is a contradiction.

Now, suppose $d_{i}^{*}=0$ and thus $\pi_{i}\left(x^{*}, d^{*}\right)=0$. We denote by $\bar{y}=\left(x_{i}^{*}, t_{i}-\sigma, x_{-i}^{*}, d_{-i}^{*}\right)$ the strategy profile in which player $i$ chooses her demand equal to $t_{i}-\sigma$ instead. Note that $\pi_{i}(\bar{y})=M\left(t_{i}-\sigma\right)-\sum_{r \in x_{i}^{*}} c_{r}\left(\ell_{r}(\bar{y})\right)$. Using that in equilibrium $d_{i}^{*}<t_{i}+\sigma$, we obtain $\pi_{i}(\bar{y}) \geq M\left(t_{i}-\sigma\right)-\sum_{r \in R} c_{r}(T)$, which is positive, because $M>2 \sum_{r \in R} c_{r}(T) / t_{\min }>\sum_{r \in R} c_{r}(T) /\left(t_{i}-\sigma\right)$. This is a contradiction to the assumption that $d_{i}^{*}=0$.

Finally, let us assume that $0<d_{i}^{*}<t_{i}-\sigma$. Referring to Lemma 3.1, we obtain the equality $U_{i}^{\prime}\left(d_{i}^{*}\right)=$ $\partial\left(d_{i}^{*} \sum_{r \in x_{i}} c_{r}\left(\ell\left(\bar{x}^{*}\right)\right)\right) / \partial d_{i}^{*}$. In particular,

$$
M=\sum_{r \in x_{i}}\left(c_{r}\left(\ell\left(\bar{x}^{*}\right)\right)+d_{i}^{*} c_{r}^{\prime}\left(\ell\left(\bar{x}^{*}\right)\right)\right) \leq \sum_{r \in R}\left(c_{r}(T)+\left(t_{i}+\sigma\right) \max _{\ell \in\left[t_{\min }, T\right]} c_{r}^{\prime}(\ell)\right),
$$

which contradicts $M>\sum_{r \in R}\left(c_{r}(T)+\left(t_{i}+\sigma\right) \max _{\ell \in\left[t_{\min }, T\right]} c_{r}^{\prime}(\ell)\right)$.

We are now ready to state our first main theorem.

Proposition 3.3. Let $\mathcal{M}$ be a congestion model and let $G^{w}=\left(N, X, \pi^{w}\right)$ be a corresponding weighted congestion game with the vector of demands $\left(d_{i}^{w}\right)_{i \in N}$. If $G^{w}$ does not admit a PNE, then there exists a congestion game with variable demands $G(\mathcal{M})$ to the same congestion model, that does not admit a PNE.

Proof. Let $\sigma<\min _{i \in N}\left(d_{i}^{\mathrm{w}}\right), M>1$ and let $G(\mathcal{M})^{M, \sigma}=G^{M, \sigma}$ be a parameterized congestion game (the parameters are $M, \sigma)$ with variable demands and utility functions $U_{i}^{M, \sigma}=g^{\sigma}\left((0,0),\left(d_{i}^{\mathrm{w}}, M d_{i}^{\mathrm{w}}\right)\right)$. We claim that for sufficiently small $\sigma$ and sufficiently large $M$, there is a game $G(\mathcal{M})^{M, \sigma}$ that does not admit a PNE.

First note that the set $X$ of strategy profiles in $G^{\mathrm{w}}$ equals the set of configuration profiles in $G^{M, \sigma}$. Since $G^{\mathrm{W}}$ does not admit a PNE, we can find for every strategy profile $x \in X$ a player $i(x) \in N$ and $y(x) \in X$ such that $\pi_{i(x)}^{\mathrm{w}}(x)>\pi_{i(x)}^{\mathrm{w}}(y(x))$ and $y(x)=\left(y_{i}(x), x_{-i}\right)$. Note that weighted congestion games are minimization games and, thus, player $i(x)$ improves her cost when switching from strategy $x_{i}$ to $y_{i}(x)$. Because the set $X$ of strategy profiles of $G^{\mathrm{w}}$ is finite, we obtain $\delta=\min _{x \in X}\left(\pi_{i(x)}(x)-\pi_{i(x)}(y(x))\right)>0$.

For a contradiction, assume that there is a PNE $\left(x^{*}, d^{*}\right)$ of $G^{M, \sigma}$. Assuming that $M$ is sufficiently large and referring to Lemma 3.2, we obtain $d_{i}^{*} \in\left(d_{i}^{\mathrm{W}}-\sigma, d_{i}^{\mathrm{w}}+\sigma\right)$ as a necessary condition. Recall that in 
the weighted congestion game $G^{\mathrm{w}}$, the deviation from $x^{*}$ to $y\left(x^{*}\right)$ is profitable for player $i\left(x^{*}\right)$. Writing $i$ shorthand for $i\left(x^{*}\right)$, we obtain

$$
\pi_{i}^{\mathrm{w}}\left(y\left(x^{*}\right)\right)-\pi_{i}^{\mathrm{w}}\left(x^{*}\right)=\sum_{r \in x_{i}^{*}} c_{r}\left(\ell_{r}\left(x^{*}\right)-\sum_{r \in y_{i}\left(x^{*}\right)} c_{r}\left(\ell_{r}\left(y\left(x^{*}\right)\right)=\sum_{r \in x_{i}^{*}} c_{r}\left(\sum_{j \in N: r \in x_{j}^{*}} d_{j}^{\mathrm{w}}\right)-\sum_{r \in y_{i}\left(x^{*}\right)} c_{r}\left(\sum_{j \in N: r \in y\left(x^{*}\right)_{j}} d_{j}^{\mathrm{w}}\right)>\delta .\right.\right.
$$

Said differently, the function $h: \mathbb{R}_{\geq 0}^{n} \rightarrow \mathbb{R}, d \mapsto \sum_{r \in x_{i}^{*}} c_{r}\left(\sum_{j \in N: r \in x_{j}^{*}} d_{j}\right)-\sum_{r \in y_{i}\left(x^{*}\right)} c_{r}\left(\sum_{j \in N: r \in y\left(x^{*}\right)_{j}} d_{j}\right)$ is positive for $d^{\mathrm{w}}$. Note that $h$ is continuous in every component implying that there is $\epsilon>0$ such that $h(d)=\sum_{r \in x_{i}^{*}} c_{r}\left(\sum_{j \in N: r \in x_{j}^{*}} d_{j}\right)-\sum_{r \in y_{i}\left(x^{*}\right)} c_{r}\left(\sum_{j \in N: r \in y\left(x^{*}\right)_{j}} d_{j}\right)>0$ for all $d \in \mathbb{R}_{\geq 0}^{n}$ with $\left|d_{i}-d_{i}^{\mathrm{w}}\right|<\epsilon$. We set $\sigma=\epsilon$ and regard again the game $G^{M, \sigma}$ for which $\pi_{i}\left(y\left(x^{*}\right), d^{*}\right)-\pi_{i}\left(x^{*}, d^{*}\right)=\sum_{r \in x_{i}^{*}} c_{r}\left(\ell_{r}\left(x^{*}, d^{*}\right)\right)-$ $\sum_{r \in y_{i}\left(x^{*}\right)} c_{r}\left(\ell_{r}\left(y(x), d^{*}\right)>0\right.$, since $d_{i}^{*} \in\left(d_{i}^{\mathrm{W}}-\epsilon, d_{i}^{\mathrm{W}}+\epsilon\right)$. We conclude that player $i$ deviates profitably from $\left(x^{*}, d^{*}\right)$ to $\left(y\left(x^{*}\right), d^{*}\right)$.

In [11] it is shown that for weighted congestion games, a set $C$ of (twice continuously differentiable) cost functions is consistent if and only if one of the following cases holds: (i) $C$ contains only affine functions; (ii) $C$ contains only exponential functions of type $c(\ell)=a_{c} e^{\phi \ell}+b_{c}$ for some constants $a_{c} \cdot b_{c}, \phi \in \mathbb{R}$ where $a_{c}$ and $b_{c}$ may depend on $c$ while $\phi$ is a common constant for all $c \in C$. By Proposition 3.3, we obtain the following result as an immediate corollary.

Corollary 3.4. If $C \subseteq C^{2}\left(\mathbb{R}_{\geq 0}\right)$ is consistent w.r.t. congestion game with variable demands then one of the following cases holds: (i) $C$ contains only affine functions; (ii) $C$ contains only exponential functions.

The upper result even holds when regarding only network congestion games with variable demands, see the discussion in [11]. Because we only need to consider non-negative and strictly increasing cost functions with unbounded marginal costs, for an affine cost function $c$, we can assume that $c(\ell)=a \ell+b$ with $a>0$, $b \geq 0$. For an exponential function $c(\ell)=a e^{\phi \ell}+b$ we can assume $a, \phi>0$ and $b \geq-a$. The function $c$ is called homogeneously exponential if $b=0$ and inhomogeneously exponential otherwise. Moreover, when we say that a set $C$ of functions contains only exponential functions, we assume that there is a universal constant $\phi>0$ such that every $c \in C$ can be written as $c(\ell)=a_{c} e^{\phi \ell}+b_{c}$ for some constants $a_{c}>0, b_{c} \geq-a_{c}$.

Excluding Inhomogeneously Exponential Costs. Next, we show that any inhomogenously exponential function $c(\ell)=a e^{\phi \ell}+b$ with $a, \phi>0$ and $b \neq 0$ is not consistent w.r.t. congestion games with variable demands. In particular, for every such function $c$ there is a congestion game with variable demands and cost equal to $c$ on all resources that does not admit a PNE. In order to prove this result, we first investigate congestion games with resource dependent demands. A complete characterization of the set of cost functions that is consistent w.r.t. this class of games is given. We will use a further refinement of this characterization to show that inhomogenously exponential function are not consistent w.r.t. congestion games with variable demands. Let us first define congestion games with resource dependent demands.

Definition 3.5. Let $\mathcal{M}=\left(N, F, X,\left(c_{r}\right)_{r \in R}\right)$ be a congestion model and let $\left(d_{i, r}\right)_{i \in N, r \in R}$ be a matrix of demands. The corresponding congestion game with resource dependent demands is the game $G(\mathcal{M})=(N, X, \pi)$, where $\pi$ is defined as $\pi=\chi_{i \in N} \pi_{i}, \pi_{i}(x)=\sum_{r \in x_{i}} d_{i, r} c_{r}\left(\ell_{r}(x)\right)$ and $\ell_{r}(x)=\sum_{j \in N: r \in x_{j}} d_{j, r}$.

Restricting the strategy sets to singletons, we obtain scheduling games on unrelated machines as a special case. Harks et al. [12] showed that congestion games with resource dependent and affine costs always admit a PNE. Here, we strengthen their result providing a complete characterization of cost functions that are consistent w.r.t. congestion games with resource dependent demand.

Theorem 3.6. A set $C \subseteq C^{2}\left(\mathbb{R}_{\geq 0}\right)$ of cost functions is consistent w.r.t. congestion games with resource dependent demands if and only if $C$ contains only affine functions. 


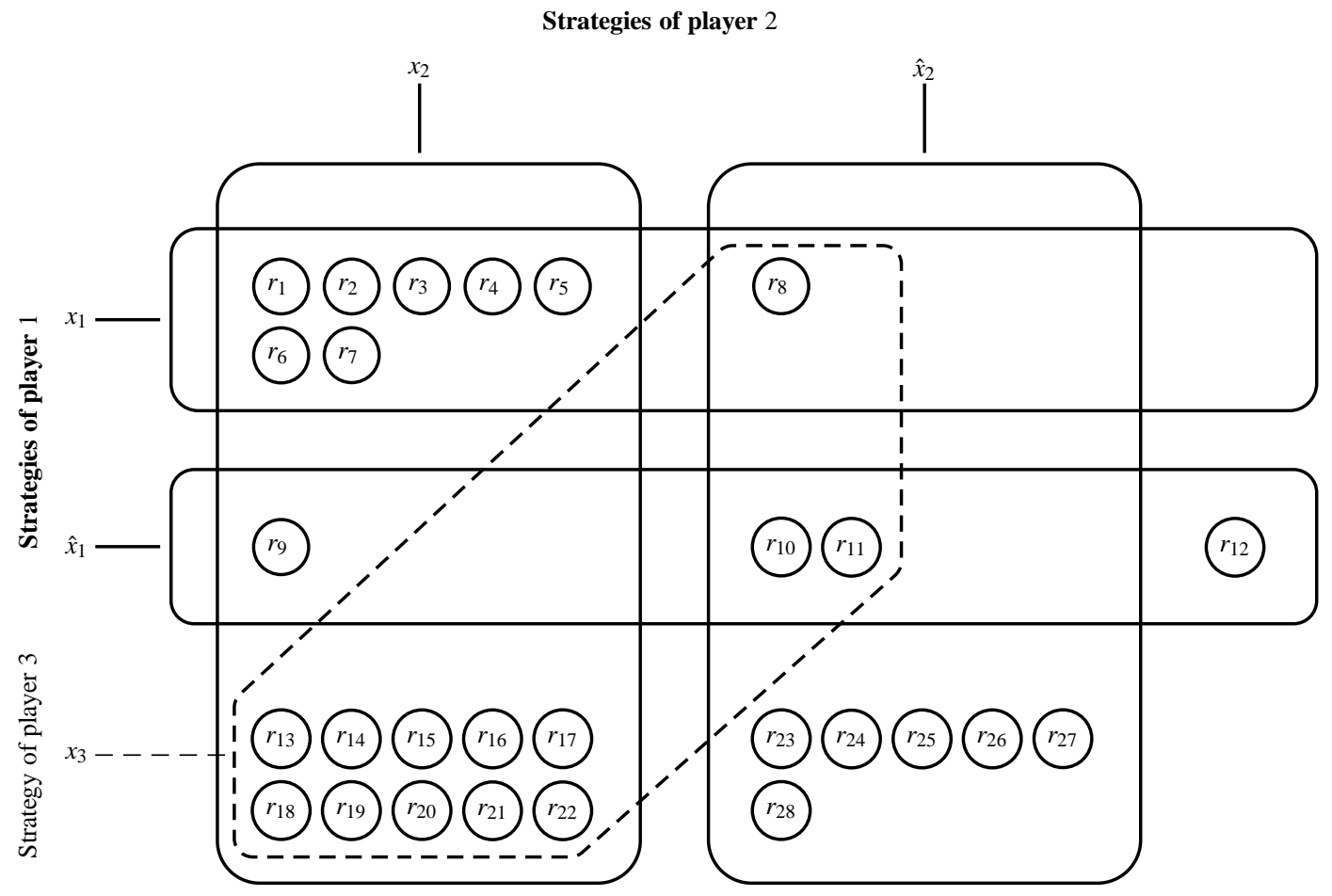

Figure 2: Strategy spaces of the three players in the game considered in the proof of Theorem 3.6 Players 1 and 2 have two strategies each, which are drawn as solid boxes. Player 3 has a single strategy which is shown as a dashed box.

Proof. Since congestion games with resource dependent demands contain weighted congestion games by setting $d_{i, r}=d_{i, r^{\prime}}$ for all $i \in N$ and $r, r^{\prime} \in R$, we know that any set of consistent cost functions $C$ contains either only affine functions or only exponential functions. So it is left to show that for every exponential cost function $c(\ell)=a e^{\phi \ell}+b$ with $a, \phi>0$ and $b \geq-a$, there is a congestion game with resource-dependent demands that does not possess a PNE.

To this end, let $c(\ell)=a e^{\phi \ell}+b$ with $a, \phi \in \mathbb{R}_{>0}$ and $b \geq-a$ be given and consider the congestion model $\mathcal{M}=\left(N, R, X,\left(c_{r}\right)_{r \in R}\right)$ with three players $N=\{1,2,3\}$ and 28 resources $R=\left\{r_{1}, \ldots, r_{28}\right\}$. The strategy spaces of player 1 and 2 contain two strategies only, that is, $X_{1}=\left\{x_{1}, \hat{x}_{1}\right\}$ and $X_{2}=\left\{x_{2}, \hat{x}_{2}\right\}$. We define $x_{1}=\left\{r_{1}, \ldots, r_{8}\right\}, \hat{x}_{1}=\left\{r_{9}, r_{10}, r_{11}, r_{12}\right\}, x_{2}=\left\{r_{1}, \ldots, r_{7}, r_{9}, r_{13}, \ldots, r_{22}\right\}, \hat{x}_{2}=\left\{r_{8}, r_{10}, r_{11}, r_{23}, \ldots, r_{28}\right\}$. Player 3 has only a single strategy $x_{3}=\left\{r_{8}, r_{13}, \ldots, r_{22}\right\}$. The strategy spaces are shown in Figure 2,

Note that the two strategies of the non-trivial players $i \in\{1,2\}$ are disjoint. The resource-dependent demands of these players are given by $d_{i, r}=\ln 2 / \phi$ for all resources $r \in x_{i}$ and $d_{i, r}=2 \ln 2 / \phi$ for all resources $r \in \hat{x}_{i}$. The demand of player 3 equals $d_{3, r}=3 / \phi$ for all $r \in R$. We calculate that

$$
\begin{aligned}
\pi_{1}\left(\hat{x}_{1}, x_{2}, x_{3}\right)-\pi_{1}\left(x_{1}, x_{2}, x_{3}\right) & =\frac{2 \ln 2}{\phi}\left(2^{2} a+b+2\left(2^{2} a+b\right)+2^{2} a+b\right)-\frac{\ln 2}{\phi}\left(7\left(2^{2} a+b\right)+8\left(2^{4} a+b\right)\right. \\
& =-124 a \frac{\ln 2}{\phi}<0 .
\end{aligned}
$$

Similarly, we obtain

$$
\begin{aligned}
& \pi_{2}\left(\hat{x}_{1}, \hat{x}_{2}, x_{3}\right)-\pi_{2}\left(\hat{x}_{1}, x_{2}, x_{3}\right)=-6 a \frac{\ln 2}{\phi}<0, \quad \pi_{1}\left(x_{1}, \hat{x}_{2}, x_{3}\right)-\pi\left(\hat{x}_{1}, \hat{x}_{2}, x_{3}\right)=-2 a \frac{\ln 2}{\phi}<0, \\
& \pi_{2}\left(x_{1}, x_{2}, x_{3}\right)-\pi_{2}\left(x_{1}, \hat{x}_{2}, x_{3}\right)=-2 a \frac{\ln 2}{\phi} .
\end{aligned}
$$


Since the game that we constructed in this proof contains only 4 strategy profiles and each profile admits a profitable deviation, we conclude that the game does not admit a PNE.

Let us call a congestion game with resource dependent demands simple if each player has a unique demand per strategy, that is, for all $i \in N$ and $x_{i} \in X_{i}$ there is $d_{x_{i}} \in \mathbb{R}_{>0}$ such that $d_{i, r}=d_{x_{i}}$ for all $r \in x_{i}$. Since the game constructed in the proof of Theorem 3.6 is simple, the statement of Theorem 3.6 is also valid for simple congestion games with resource dependent demands. In particular, for every non-affine function, there is a congestion model $\mathcal{M}=\left(N, R, X,\left(c_{r}\right)_{r \in R}\right)$ and a corresponding simple congestion game with resource dependent demands $G^{\text {rd }}=G^{\text {rd }}(\mathcal{M})$ with costs equal to $c$ on all resources that has an improvement cycle $\left(x^{1}, x^{2}, \ldots, x^{s}, x^{1}\right)$. Now consider a congestion game with variable demands $G=G(\mathcal{M})$ to the same model, where the utility of each player is constant. Clearly, the cycle $\left(\left(x^{1}, d^{1}\right),\left(x^{2}, d^{2}\right), \ldots,\left(x^{s}, d^{s}\right),\left(x^{1}, d^{1}\right)\right)$, where $d_{i}^{k}$ equals the fixed demand of player $i$ in the congestion game with resource dependent demand is also an improvement cycle for $G$. We have established the following.

Proposition 3.7. For a set $C \subseteq C^{2}\left(\mathbb{R}_{\geq 0}\right)$ the following three are equivalent: (i) $C$ contains only affine functions; (ii) $C$ is FIP consistent w.r.t. congestion games with variable demands; (iii) $C$ is consistent w.r.t. congestion games with resource dependent demands.

Note that for any non-affine cost function $c$, there is a congestion game with variable demands and costs equal to $c$ with an improvement cycle. However, there might be PNE outside that cycle.

Our basic idea for a complete characterization is the following. Let $c$ be an arbitrary exponential function. Using Theorem 3.6 there is a simple congestion game with resource dependent demands $G^{r d}$ that does not admit a PNE. We want to construct a congestion game with variable demands $G$ where players' have access on the same resources. We design the players' concave and non-decreasing utility functions such that for any $\operatorname{PNE}\left(x_{i}, x_{-i}^{*}, d_{i}^{*}, d_{-i}^{*}\right)$ in which player $i$ plays configuration $x_{i}$, the equilibrium demand $d_{i}^{*}$ is close to $d_{x_{i}}$. The key to make this idea work is to introduce additional resources so as to ensure that the players' marginal costs enforce these equilibrium demands. As it turns out, this manipulation can only be done for the case of inhomogenously exponential cost functions. To illustrate this idea, we give an example. If we add two resources with $\operatorname{cost} c(\ell)=e^{x}+1$ to a players' strategy who plays a demand equal to 1 , her costs are increased by $2 e+2$ while her marginal costs are increased by $4 e+2$. If we instead add one resource with the same cost and a trivial player who plays a demand equal to $\ln \left(2+e^{-1}\right)$, then her costs are increased by $2 e+2$ as well, but her marginal costs are increased by $4 e+3$. In this fashion, we can increase the marginal costs of one configurations more than in some other configuration while leaving their differences in costs constant. Since increasing the marginal cost of a configuration, decreases the equilibrium demand, the key challenge is to manipulate the players' marginal costs in order to enforce them play the right equilibrium demands. Before we will develop this idea in the proof of Proposition 3.9, we need the following technical lemma which is a strengthening of Theorem 3.6 .

Lemma 3.8. Let $\Delta>0$ be arbitrary. Theorem 3.6 holds even for simple congestion games with resource dependent demands, where for each player one of the following holds: (i) Player $i$ has exactly one strategy, that is, $X_{i}=\left\{x_{i}\right\}$ with $x_{i} \subseteq R$; (ii) Player $i$ has exactly two strategies that she uses with demands that differ by $\Delta$, that is, $X_{i}=\left\{x_{i}, \hat{x}\right\}$ and $\left|d_{x_{i}}-d_{\hat{x}_{i}}\right|=\Delta$ with $x_{i}, \hat{x}_{i} \subseteq R$.

Proof. To obtain this more general result, we need a more involved construction compared to that of the proof of Theorem 3.6. Let $c(\ell)=a e^{\phi \ell}+b$ with $a, \phi \in \mathbb{R}_{>0}$ and $b \geq-a$ and consider for $p, q \in \mathbb{N}_{>0}$ the congestion model $\mathcal{M}=\left(N, R, X,\left(c_{r}\right)_{r \in R}\right)$ with 5 players and $6 q+p$ resources

$$
R=\left\{r_{1}, \ldots, r_{p}, s_{1}, \ldots, s_{2 q}, v_{1}, \ldots, v_{2 q}, w_{1}, \ldots, w_{2 q}\right\} .
$$


Only the first two players $i \in\{1,2\}$ have two strategies and will be called non-trivial players. Their strategy spaces are given by

$$
\begin{array}{llll}
X_{1}=\left\{x_{1}, \hat{x}_{1}\right\}, & \text { where } & x_{1}=\left\{r_{1}, \ldots, r_{p}, s_{1}, \ldots, s_{2 q}\right\}, & \hat{x}_{1}=\left\{v_{1}, \ldots, v_{2 q}\right\}, \\
X_{2}=\left\{x_{2}, \hat{x}_{2}\right\}, & \text { where } & x_{2}=\left\{r_{1}, \ldots, r_{p}, w_{1}, \ldots, w_{2 q}\right\}, & \hat{x}_{2}=\left\{s_{1}, \ldots, s_{2 q}\right\} .
\end{array}
$$

The players 3, 4, and 5 have only one strategy and serve only to increase the costs on some of the resources. They will be called trivial players. Their strategies are given by $X_{3}=\left\{x_{3}\right\}=\left\{\left\{r_{1}, \ldots, r_{p}\right\}\right\}, X_{4}=\left\{x_{4}\right\}=$ $\left\{s_{1}, \ldots, s_{2 q}\right\}, X_{5}=\left\{x_{5}\right\}=\left\{\left\{v_{1}, \ldots, v_{2 q}\right\}\right\}$. The strategy spaces are shown in Figure 3 (a). Observe that the strategies of the two non-trivial players are disjoint. For the non-trivial players $i \in\{1,2\}$, we set $d_{i, r}=2 \frac{\ln 2}{\phi}$ for all $r \in x_{i}$ and $d_{i, r}=\left(2+\frac{p}{q}\right) \frac{\ln 2}{\phi}$ for all $r \in \hat{x}_{i}$.

Assume that the demand of the non-trivial players 3, 4, and 5 are given by $d_{3, r}=\ln \left(a_{3}\right) / \phi, d_{4, r}=$ $\ln \left(a_{4}\right) / \phi$, and $d_{5, r}=\ln \left(a_{5}\right) / \phi$ for all $r \in R$, where $a_{3}, a_{4}, a_{5}>1$ are parameters. We will carefully choose the parameters $a_{3}, a_{4}$, and $a_{5}$ such that

$$
\gamma=\left(\left(x_{1}, x_{2}, x_{3}, x_{4}, x_{5}\right),\left(\hat{x}_{1}, x_{2}, x_{3}, x_{4}, x_{5}\right),\left(\hat{x}_{1}, \hat{x}_{2}, x_{3}, x_{4}, x_{5}\right),\left(x_{1}, \hat{x}_{2}, x_{3}, x_{4}, x_{5}\right),\left(x_{1}, x_{2}, x_{3}, x_{4}, x_{5}\right)\right)
$$

is an improvement cycle. Calculating the costs of the respective deviating non-trivial player in $\gamma$, we obtain the following necessary and sufficient conditions

$$
\begin{aligned}
2^{5} p a_{3}+2^{4} q a_{4} & >(2+p / q) 2^{3+p / q} q a_{5} \\
2^{3} p a_{3}+2^{4} q & >(2+p / q) 2^{3+p / q} q a_{4} \\
(2+p / q) 2^{3+p / q} q a_{5} & >2^{3} p a_{3}+2^{6+p / q} q a_{4} \\
(2+p / q) 2^{5+p / q} q a_{4} & >2^{5} p a_{3}+2^{4} q .
\end{aligned}
$$

First, note that the right hand side of inequality (G1) and the left hand side of inequality (G3) are equal. This is due to the fact that the second strategy of the first player can be seen as an outside option of player 1 since none of the resources in that strategy is contained in one of the strategies of the other non-trivial player 2. Thus it is sufficient to consider the inequality

$$
\begin{aligned}
2^{5} p a_{3} & +2^{4} q a_{4}>2^{3} p a_{3}+2^{6+p / q} q a_{4} \\
& \Leftrightarrow \quad p a_{3}>\frac{8}{3} 2^{p / q} q a_{4}-\frac{2}{3} q a_{4}
\end{aligned}
$$

instead of (G1) and (G3). Once this conditions is satisfied one can choose $a_{5}$ appropriately. Inequalities (G2) and (G4) give rise to

$$
\begin{aligned}
p a_{3} & >2^{p / q}(2 q+p) a_{4}-2 q, \\
2^{p / q}(2 q+p) a_{4}-q / 2 & >p a_{3},
\end{aligned}
$$

respectively. It is left to show that inequalities [G1'], [G2'), and (G4') can be satisfied simultaneously. To this end, observe that the function $f(x)=2^{x}\left(x-\frac{2}{3}\right)+\frac{2}{3}$ is strictly increasing for $x \geq 0$. As $f(0)=0$, the function $f$ is strictly positive for every $x>0$. This implies that the inequality $2^{p / q}\left(\frac{p}{q}-\frac{2}{3}\right)+\frac{2}{3}>0$ holds for all $p, q \in \mathbb{N}_{>0}$

We define $a_{4}>\max \left\{1,1 /\left(2^{p / q}\left(\frac{p}{q}-\frac{2}{3}\right)+\frac{2}{3}\right\}\right.$ and $a_{3}=2^{p / q}(2 q / p+1) a_{4}-\frac{q}{p}$. Since $q>0$, clearly $a_{3}$ and $a_{4}$ satisfy (G2') and (G4). As for (G1') note that

$$
p a_{3}-\frac{8}{3} 2^{p / q} q a_{4}+\frac{2}{3} q a_{4}=a_{4}\left(\frac{2}{3} q-\frac{2}{3} 2^{p / q} p-2^{p / q} p\right)-q>0 .
$$


We set $a_{5}=\frac{1}{2(2+p / q) 2^{3+p / q} q}\left(2^{5} p a_{3}+2^{4} q a_{4}-2^{3} p a_{3}-2^{6+p / q} q a_{4}\right)=\frac{1}{(2+p / q) 2^{3+p / q} q}\left(12 p a_{3}+\left(2^{3}-2^{5+p / q}\right) q a_{4}\right)$. Clearly, $a_{5}$ satisfies (G1) and (G3). It is left to show, that $a_{3}, a_{4}$, and $a_{5}$ are not smaller than 1 . Since $a_{4}>1$ by definition, (G1) implies $a_{3}>\frac{q}{p}\left(\frac{8}{3} 2^{p / q}-\frac{2}{3}\right)>1$. As for $a_{5}$, inequality (G3) gives rise to

$$
a_{5}>\frac{p a_{3}}{\left(2+\frac{p}{q}\right) q 2^{p / q}}+\frac{8 a_{4}}{\left(2+\frac{p}{q}\right) q}=a_{4}-\frac{q}{\left(2+\frac{p}{q}\right) q 2^{p / q}}+\frac{8 a_{4}}{\left(2+\frac{p}{q}\right) q}>1+\frac{2^{3+p / q} q-1}{\left(2+\frac{p}{q}\right) 2^{p / q}}>1
$$

Thus, we have found $a_{3}, a_{4}, a_{5}>1$ such that $\gamma$ is an improvement cycle, establishing that the corresponding game does not admit a PNE.

We are now ready for the main result of this section.

Proposition 3.9. Any inhomogenously exponential function is not consistent w.r.t. congestion games with variable demands.

Proof. Let $c(\ell)=a e^{\phi \ell}+b$ with $a, \phi>0$ and $b \geq-a, b \neq 0$ be an inhomogeneously exponential function. We first assume that $\phi=1$ and $b>0$. In the concluding remarks of this proof, we sketch how the arguments can be adapted to work with arbitrary $\phi>0$ and $-a \leq b<0$.

As shown in Lemma 3.8, for every $p, q \in \mathbb{N}_{>0}$ there is a congestion model $\mathcal{M}=\left(N, R, X,\left(c_{r}\right)_{r \in R}\right)$ with $p+6 q$ resources, 5 players and cost functions $c_{r}=c$ for all $r \in R$ such that there is a corresponding congestion game with resource dependent demands $G^{\mathrm{rd}}=\left(N, X, \pi^{\mathrm{rd}}\right)$ that does not admit a PNE. The game $G^{\text {rd }}$ can be constructed to have the following additional properties: The set of players $N^{\text {rd }}$ is partitioned into two non-trivial players $N_{\mathrm{nt}}$ and three trivial players $N_{\mathrm{t}}$. Every trivial player $i \in N_{\mathrm{t}}$ has a unique strategy $X_{i}=\left\{x_{i}\right\}$ and a unique demand $t_{i}=d_{i, r}$ for all $r \in R$. Every non-trivial player $i \in N_{\text {nt }}$ has exactly two disjoint strategies $X_{i}=\left\{x_{i}, \hat{x}_{i}\right\}$ and exactly two demands $t_{i}=2$ and $\hat{t}_{i}=2+p / q$, such that $d_{i, r}=t_{i}$ for all $r \in x_{i}$ and $d_{i, r}=\hat{t}_{i}$ for all $r \in \hat{x}_{i}$. For notational convenience, we use the convention $t_{i}=\hat{t}_{i}$ for every trivial player $i \in N_{\mathrm{t}}^{\mathrm{rd}}$.

Based on the game $G^{\text {rd }}$ described above, we want to construct a congestion game with variable demands $G$ that does not admit a PNE. Our construction is based on carefully designing the players' utility function. In fact, the players' utilities should offer them an incentive to play (almost) the same demands as in the congestion game with resource dependent demands. That is, we want to assure that in any PNE of $G$ (if it exists) if player $i$ uses the configuration $x_{i}$, then her demand is close to $t_{i}$. In contrast, if player $i$ plays $\hat{x}_{i}$, then her demand is close to $\hat{t}_{i}$.

As shown in Lemma 3.2, if $M_{i}$ is sufficiently large and the utility of the trivial player $i \in N_{\mathrm{t}}$ is given by the smoothed 2-wise linear function $U_{i}=g^{\sigma}\left((0,0),\left(t_{i}, M_{i} t_{i}\right)\right)$, then $d_{i}^{*} \in\left(t_{i}-\sigma, t_{i}+\sigma\right)$ in every $\operatorname{PNE}\left(x^{*}, d^{*}\right)$ of $G$.

For the non-trivial players, we will use a similar idea. We define their utilities such that $d_{i}^{*} \in\left(t_{i}-\sigma, t_{i}+\sigma\right)$ for every $\operatorname{PNE}\left(\left(x_{i}, x_{-i}^{*}\right), d^{*}\right)$ in which player $i$ plays her first configuration $x_{i}$ and $d_{i}^{*} \in\left(\hat{t}_{i}-\sigma, \hat{t}_{i}+\sigma\right)$ for every PNE $\left(\left(\hat{x}_{i}, x_{-i}^{*}\right), d^{*}\right)$ in which player $i$ plays her second configuration $\hat{x}_{i}$. We note the subtle disambiguation between strategies in congestion games (with resources-dependent demands) and strategies in congestion games with variable demands. While a strategy in a congestion game is a subset of resources, a strategy in a congestion game with variable demands is a tuple of a subset of resources and a demand. To distinguish between both, we call the sets $x_{i} \in X_{i} \subseteq 2^{R}$ strategies when speaking of the congestion game with resource dependent demand and configurations when dealing with the congestion game with variable demand.

To enforce the right demands in the congestion game with variable demands, we will enhance the underlying congestion model. We introduce new resources (parameterized by $m, \hat{m}$ ) and additional players in Step 2. In Step 3, we will choose the parameters $p, q, m, \hat{m} \in \mathbb{N}_{>0}$ and some $\epsilon>0$. The utility functions of the non-trivial players will be defined in Step 4. Finally, in Step 5 we show that the thus constructed congestion game with variable demand does not admit a PNE. 


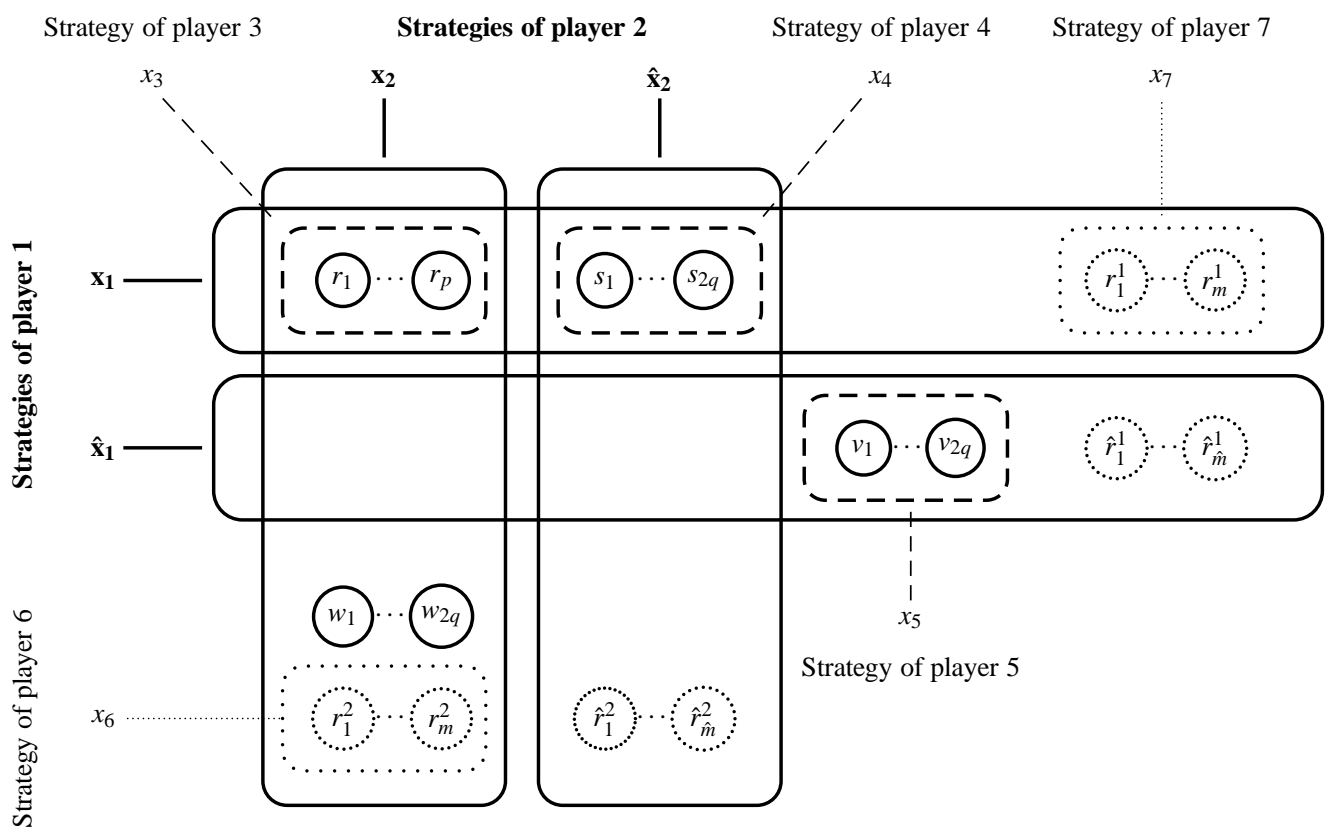

(a) Non-network congestion game

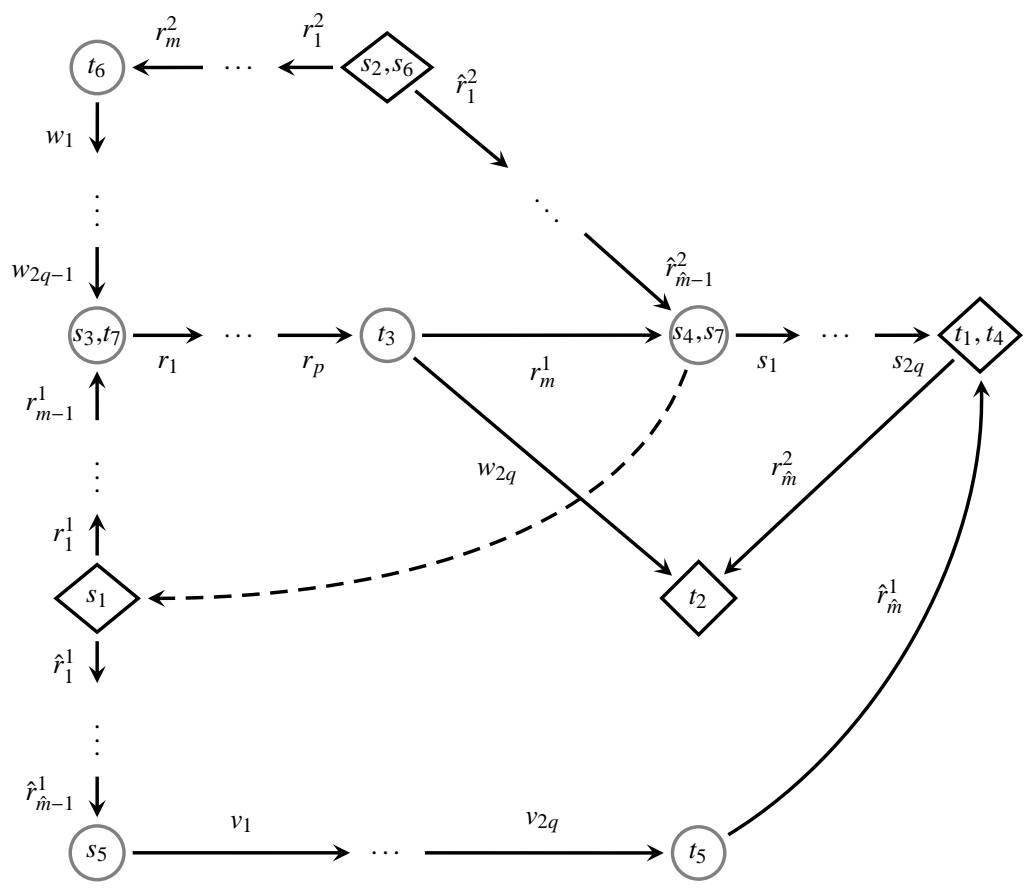

(b) Network congestion game

Figure 3: Strategy spaces of the of the congestion games constructed in the proof of Lemma3.9 in their non-network and network representations. In the non-network representation (a) the non-trivial players 1 and 2 each have two strategies shown by boxes with solid lines while the trivial players 3,4 and 5 each have a single strategy drawn as dashed boxes. Omitting the dashed resources as well as the new trivial players 6 and 7 one obtains the congestion model constructed in Lemma 3.8 on which the proof of Lemma 3.9 is based. In the network representation (b) resources correspond to the edges of the graph. Each player $i$ is associated with a pair $\left(s_{i}, t_{i}\right)$ of nodes. The set of strategies of each player equals the set of her directed $\left(s_{i}, t_{i}\right)$-paths. By adding sufficiently many resources to the path that is indicated by the dashed line, the set of every players undominated non of the non-trivial players will use this path in equilibrium. 
Step 2: Enhancing the congestion model. For every non-trivial player $i \in N_{\text {nt }}$ we add $m \in \mathbb{N}$ new resources to $i$ 's first strategy $x_{i}$ and $\hat{m} \in \mathbb{N}$ resources to $i$ 's second strategy $\hat{x}_{i}$. In addition, the $2 m$ new resources added to $x_{1}$ and $x_{2}$, respectively, shall be used by two new trivial players. Technically, we introduce a new congestion model $\mathcal{M}^{\prime}=\left(N^{\prime}, R^{\prime}, X^{\prime},\left(c_{r}\right)_{r \in R^{\prime}}\right)$. The new set of players contains two new trivial players. Thus, we define $N_{\mathrm{t}}^{\prime}=N_{\mathrm{t}} \cup\{6,7\}, N_{\mathrm{nt}}^{\prime}=N_{\mathrm{nt}}$, and $N^{\prime}=N_{\mathrm{nt}}^{\prime} \cup N_{\mathrm{t}}^{\prime}$. For every non-trivial player $i \in N_{\mathrm{nt}}^{\prime}$, we set $x_{i}^{\prime}=x_{i} \cup\left\{r_{1}^{i}, \ldots, r_{m}^{i}\right\}$ and $\hat{x}_{i}^{\prime}=\hat{x}_{i} \cup\left\{\hat{r}_{1}^{i}, \ldots, \hat{r}_{\hat{m}}^{i}\right\}$. Note that the new set of resources contains $p+6 q+2 m+2 \hat{m}$ resources, so

$$
R^{\prime}=\left\{r_{1}, \ldots, r_{p}, s_{1}, \ldots, s_{2 q}, v_{1}, \ldots, v_{s q}, w_{1}, \ldots, w_{2 q}, r_{1}^{1}, \ldots, r_{m}^{1}, \hat{r}_{1}^{1}, \ldots, \hat{r}_{\hat{m}}^{1}, r_{1}^{2}, \ldots, r_{m}^{2}, \hat{r}_{1}^{2}, \ldots, \hat{r}_{\hat{m}}^{2}\right\} .
$$

The (old) trivial players 3, 4, and 5, have the same strategies as in $G^{\text {rd }}$. The new trivial player 6 has a single strategy $x_{6}^{\prime}=\left\{r_{1}^{1}, \ldots, r_{m}^{1}\right\}$, the single strategy of player 7 is $x_{7}^{\prime}=\left\{r_{1}^{2}, \ldots, r_{m}^{2}\right\}$. For some $\epsilon>0$ to be defined later, we want these two players to play a demand close to $t_{6}=t_{7}=p / q+\ln \left(\frac{3+\epsilon+p / q}{3}\right)+\ln \left(\frac{\hat{m}}{m}\right)$ in equilibrium. As we already argued for the other trivial players 3,4 , and 5 choosing the right smoothed 2-wise linear function for players 6 and 7 their equilibrium demands can be restricted to $d_{6}^{*} \in\left(t_{6}-\sigma, t_{6}+\sigma\right)$ and $d_{7}^{*} \in\left(t_{7}-\sigma, t_{7}+\sigma\right)$, respectively.

Observe that the marginal utility of each player $i \in N^{\prime}$ is zero for demands $d_{i} \geq \hat{t}_{i}+1 / 2$. Hence, in any $\operatorname{PNE}\left(x^{\prime *}, d^{*}\right)$, every player $i \in N^{\prime}$ will use a demand $d_{i}^{*}<\hat{t}_{i}+1 / 2$. Let us now consider a non-trivial player $j \in N_{\mathrm{nt}}^{\prime}$. We observe that $j$ 's marginal cost $\mathrm{MC}_{j}\left(x_{j}^{\prime}, x_{-j}^{*}, t_{j}, d_{-j}^{*}\right)=\partial t_{j} \sum_{r \in x_{j}} c_{r}\left(\ell_{r}\left(x_{j}^{\prime}, x_{-j}^{*}, t_{j}, d_{-j}^{*}\right)\right) / \partial t_{j}$ when playing her first configuration $x_{j}^{\prime}$ and her first target demand $t_{j}=2$ can be bounded from below by

$$
\begin{aligned}
\operatorname{MC}_{j}\left(x_{j}^{\prime}, x_{-j}^{*}, t_{j}, d_{-j}^{*}\right) & =3 \sum_{r \in x_{j}} a e^{\ell_{r}\left(x_{j}^{\prime}, x_{-j}^{*}, t_{j}, d_{-j}^{*}\right)}+\left|x_{j}\right| b+a \hat{m}\left(3+\epsilon+\frac{p}{q}\right) e^{2+p / q}+m b \\
& >a\left(3+\frac{p}{q}\right) \hat{m} e^{2+p / q}+a \hat{m} \epsilon e^{2+p / q}+m b .
\end{aligned}
$$

for all $x_{-j}^{* *} \in X_{-j}^{\prime}$ and $d_{-j}^{*} \in X_{i \neq j}\left[0, \hat{t}_{i}+1 / 2\right]$. Note that the summation is over $r \in x_{i}$, that is, we do not sum over the $m$ newly introduced resources. Assuming that no player uses a non-optimal demand (which gives a marginal utility equal to zero) and that $\sigma \leq 1$, player $j$ 's marginal costs when playing her first strategy and her first target demand can also be bounded from above by

$$
\mathrm{MC}_{j}\left(x_{j}^{\prime}, x_{-j}^{* *}, t_{j}, d_{-j}^{*}\right) \leq \omega_{j}+a \hat{m}\left(3+\epsilon+\frac{p}{q}\right) e^{2+p / q}+m b
$$

where $\omega_{j}=3 \sum_{r \in R} a e^{4+2 p / q+\sum_{j=3, \ldots, 7} t_{j}+7}+|R| b$. Analogously, in every equilibrium $j$ 's marginal cost when playing her second configuration $\hat{x}_{j}^{\prime}$ and her second target demand $\hat{t}_{j}=2+p / q$ can be bounded from above by

$$
\begin{aligned}
\operatorname{MC}_{j}\left(\hat{x}_{j}^{\prime}, x_{-j}^{\prime *}, \hat{t}_{j}, d_{-j}^{*}\right) & =\left(3+\frac{p}{q}\right) \sum_{r \in \hat{x}_{j}} a e^{\ell_{r}\left(\hat{x}_{j}, x_{-j}^{\prime *}, \hat{t}_{j}, d_{-j}^{*}\right)}+\left|\hat{x}_{j}\right| b+\left(3+\frac{p}{q}\right) a \hat{m} e^{2+p / q}+\hat{m} b \\
& <\omega_{j}+a\left(3+\frac{p}{q}\right) \hat{m} e^{2+p / q}+\hat{m} b .
\end{aligned}
$$

for all $x_{-j}^{\prime *} \in X_{-j}^{\prime}$ and $d_{-j}^{*} \in X_{i \neq j}\left[0, \hat{t}_{i}+1 / 2\right]$.

So $j$ 's marginal cost when playing her first configuration $x_{j}^{\prime}$ with low demand $t_{j}=2$ can be bounded from below by $B=a \hat{m}\left(3+\frac{p}{q}\right) e^{2+p / q}+a \hat{m} \epsilon e^{2+p / q}+b m$ and her marginal cost when playing her second configuration $\hat{x}_{j}^{\prime}$ with high demand $\hat{t}_{j}=2+p / q$ can be bounded form above by $\hat{B}=\omega_{j}+a \hat{m}(3+p / q) e^{2+p / q}+\hat{m} b$. Moreover, 
note that $j$ 's actual cost when playing her first configuration $x_{j}^{\prime}$ with low demand $t_{j}$ has been increased by $\frac{2}{3}(3+\epsilon+p / q) a \hat{m} e^{2+p / q}+2 b m$ by adding $m$ additional resources. In contrast, the cost when playing her second configuration $\hat{x}_{j}^{\prime}$ with high demand $\hat{t}_{j}$ has been increased by $(2+p / q) a \hat{m} e^{2+p / q}+(2+p / q) b \hat{m}$. We want that the slope $\tau_{j}$ of player $j$ 's utility between $t_{j}$ and $\hat{t}_{j}$ is such that $B>\tau_{j}>\hat{B}$ in order to give player $j$ the incentive to play $x_{j}^{\prime}$ only together with demand $t_{j}$ and $\hat{x}_{j}^{\prime}$ only together with demand $\hat{t}_{j}$, respectively.

As we want to cancel out the difference in utility $U_{j}\left(\hat{t}_{j}\right)-U_{j}\left(t_{j}\right) \approx \frac{p}{q} \tau_{j}$ by the additional costs, we require

$$
\frac{p}{q} \tau_{j}=\left(2+\frac{p}{q}\right) a \hat{m} e^{2+p / q}+\left(2+\frac{p}{q}\right) b \hat{m}-\frac{2}{3}\left(3+\epsilon+\frac{p}{q}\right) a \hat{m} e^{2+p / q}-2 b m .
$$

We proceed showing how to choose $p, q, m, \hat{m}$ and $\epsilon$ in order to satisfy $B>\tau_{j}>\hat{B}$ and (5).

Step 3: Choosing the parameters $\mathbf{p}, \mathbf{q}, \mathbf{m}, \hat{\mathbf{m}} \in \mathbb{N}_{>\mathbf{0}}$ and $\epsilon>\mathbf{0}$. Introducing $x=p / q$, we want to solve

$$
a \hat{m}(3+x) e^{2+x}+a \hat{m} \epsilon e^{2+x}-b(\hat{m}-m)>\left(\frac{1}{3}-\frac{2 \epsilon}{3 x}\right) a \hat{m} e^{2+x}+\frac{2 b}{x}(\hat{m}-m)>a \hat{m}(3+x) e^{2+x}+\omega_{i}
$$

Defining $\epsilon=\frac{2 b}{a} e^{-2-x}, m=1$ and dividing by $\hat{m}$ we get

$$
a(3+x) e^{2+x}+b+\frac{b}{\hat{m}}>\frac{2}{3} b x^{-1}+\frac{1}{3} a e^{2+x}-\frac{2 b}{x \hat{m}}>a(3+x) e^{2+x}+\frac{\omega_{i}}{\hat{m}} .
$$

The function $f(x)=\frac{2}{3} b x^{-1}+\frac{1}{3} a e^{2+x}-a(3+x) e^{2+x}$ is continuous with respect to $x$. Moreover, $f$ goes to $\infty$ as $x \rightarrow 0$ and goes to $-\infty$ as $x \rightarrow \infty$. In Particular, there is a rational $x=p / q$ such that $b>f(x)>0$. Hence, $x$ solves (7) for sufficiently large $\hat{m}$.

For the sequel of this proof, let us fix $p, q, m$ and $\hat{m}$ such that they solve (6). We continue defining the non-trivial players' utilities.

Step 4: Defining the non-trivial players' utilities. For every non-trivial player $j \in N_{\mathrm{nt}}^{\prime}$ we define the utility function $U_{j}$ as the 3-wise linear function $U_{j}=g^{\sigma}\left((0,0),\left(t_{j}, M_{j} t_{j}\right),\left(\hat{t}_{j}, M_{j} t_{j}+\tau_{j}\left(\hat{t}_{j}-t_{j}\right)\right)\right)$ with $\tau_{j}=\left(\frac{1}{3}-\frac{2 \epsilon}{x}\right) a \hat{m} e^{2+x}+\frac{2 b}{x}(\hat{m}-m)$ and $M_{j}>\omega_{j}+\hat{m}(3+\epsilon+p / q) e^{2+p / q}+m b$. As marginal costs and marginal utilities coincide in any equilibrium, we obtain that $d_{j}^{*} \in\left(t_{j}-\sigma, t_{j}+\sigma\right)$ for every $\operatorname{PNE}\left(\left(x_{j}^{\prime}, x_{-j}^{*}\right), d^{*}\right)$ in which player $j$ plays her first configuration $x_{j}^{\prime}$ and $d_{j}^{*} \in\left(\hat{t}_{j}-\sigma, \hat{t}_{j}+\sigma\right)$ for every $\operatorname{PNE}\left(\left(\hat{x}_{j}^{\prime}, x_{-j}^{*}\right), d^{*}\right)$ in which player $j$ plays her second configuration $\hat{x}_{j}^{\prime}$. We finish the proof showing that we can find a sufficiently small $\sigma$ for which no PNE of $G$ exists.

Step 5: Choosing $\sigma$ such that $G$ does not admit a PNE. For contradiction, assume that for every $\sigma>0$ there is a PNE $\bar{x}=\left(x^{\prime *}, d^{*}\right)$ of $G$. Let us first consider the case $x_{1}^{\prime *}=x_{1}$ and $x_{2}^{\prime *}=x_{2}$, that is both non-trivial players use their first strategy. As we argued in the last paragraph, we can deduce $d_{i}^{*} \in\left(t_{i}-\sigma, t_{i}+\sigma\right)$ for all $i \in N^{\prime}$ as a necessary condition on the equilibrium demand.

We claim that player 1 improves her utility when switching from $\bar{x}=\left(x_{1}^{\prime}, d_{1}^{*}\right)$ to $\bar{y}=\left(\hat{x}_{1}^{\prime}, \hat{t}_{1}\right)$. Note that

$$
\begin{aligned}
\pi_{1}(\bar{y})-\pi_{1}(\bar{x})= & U_{1}\left(\hat{t}_{1}\right)-\sum_{r \in \hat{x}_{1}} c_{r}\left(\ell_{r}(\bar{y})\right)-a \hat{m}\left(2+\frac{p}{q}\right) e^{2+p / q}-b \hat{m}\left(2+\frac{p}{q}\right) \\
& -\left(U_{1}\left(d_{1}^{*}\right)-\sum_{r \in x_{1}} c_{r}\left(\ell_{r}(\bar{x})-a \hat{m}\left(2+\frac{2 \epsilon}{3}+\frac{2 p}{3 q}\right) e^{2+p / q}-2 b m\right)\right. \\
= & U_{1}\left(\hat{t}_{1}\right)-U_{1}\left(d_{1}^{*}\right)-\left(\frac{p}{3 q}-\frac{2 \epsilon}{3}\right) a \hat{m} e^{2+p / q}-2 b(\hat{m}-m)-\frac{2 p}{q} b \hat{m}+\sum_{r \in x_{1}} c_{r}\left(\ell_{r}(\bar{x})-\sum_{r \in \hat{x}_{1}} c_{r}\left(\ell_{r}(\bar{y})\right)\right.
\end{aligned}
$$


Since in the congestion game with resource dependent demands $G^{\mathrm{rd}}$, the deviation from $\left(x_{1}, x_{2}, \ldots, x_{5}\right)$ to $\left(\hat{x}_{1}, x_{2}, \ldots, x_{5}\right)$ is profitable for player 1 , there is $\delta>0$ such that the sum $\sum_{r \in x_{1}} c_{r}\left(\ell_{r}(\bar{x})-\sum_{r \in \hat{x}_{1}} c_{r}\left(\ell_{r}(\bar{y})\right)>\right.$ $\delta>0$. Moreover, note that player 1's utility function is chosen such that $U_{1}\left(\hat{t}_{1}\right)-U_{1}\left(d_{1}^{*}\right)$ goes to $\frac{p}{q} \tau=$ $\left(\frac{p}{3 q}-\frac{2 \epsilon}{3}\right) a \hat{m} e^{2+x}+2 b(\hat{m}-m)+\frac{2 p}{q} b \hat{m}$ as $\sigma$ goes to zero. We conclude that also in the congestion game with variable demands, player 1 deviates profitable from $\bar{x}=\left(x_{1}^{\prime}, d_{1}^{*}\right)$ to $\bar{y}=\left(\hat{x}_{1}^{\prime}, \hat{t}_{1}\right)$ for $\sigma$ sufficiently small. This contradicts our assumption and we derive that $G$ does not admit a PNE.

Concluding remarks. With the above construction we have established that for every cost function $c(\ell)=$ $a e^{\phi \ell}+b$ with $\phi=1$ and $b>0$, there is a congestion game with variable demands and cost equal to $c$ on all resources that does not possess a PNE.

We sketch the argumentation for $-a \leq b<0$. The construction is the same except that in Step 3, we require that $\hat{m}<m$ and we set the target demands of the new trivial players to $t_{6}=t_{7}=p / q+\ln \left(\frac{3+\epsilon+p / q}{3}\right)>0$. It is easy to verify that the inequalities (2), (3), and (4) stay valid. Then, as $b<0$ and $\hat{m}-m<0$, we can solve inequality (6) in Step 3 with the same argument as before. Steps 4 and 5 remain as in the original proof.

For arbitrary $\phi>0$, we scale the target demands $t_{i}$ and $\hat{t}_{i}$ of every player by $1 / \phi$.

Remark 3.10. The above result also holds for network congestion games, see the construction in Figure 3 (b).

Below, we can restrict our search space for consistent cost functions to affine functions and homogeneously exponential functions. In the next section, we will show that both classes of functions are consistent.

\section{Consistent Cost Functions}

Affine Functions. We will first show below that games with affine cost functions are exact potential games and, thus, they possess a PNE and the $\alpha$-FIP for every $\alpha>0$.

Proposition 4.1. Let $G$ be a congestion game with variable demands and affine cost functions $\left(c_{r}\right)_{r \in R}$. Then, the function $P(x, d)=\sum_{i \in N} U_{i}\left(d_{i}\right)-\sum_{r \in x_{i}} c_{r}\left(\sum_{j \in\{1, \ldots, i\}: r \in x_{j}} d_{j}\right) d_{i}$ is an exact potential function. Thus, $G$ possesses a PNE and the $\alpha$-FIP for every $\alpha>0$.

Proof. We consider affine cost functions, that is, $c_{r}(\ell)=a_{r} \ell+b_{r}, a_{r}>0, b_{r} \geq 0$. We define the function $c_{r}^{\leq i}(x, d)=c_{r}\left(\sum_{j \in\{1, \ldots, i\}: r \in x_{j}} d_{j}\right)$ and rewrite $P(x, d)$ as $P(x, d)=\sum_{i \in N} P_{i}(x, d)$, where $P_{i}(x, d)=$ $U_{i}\left(d_{i}\right)-\sum_{r \in x_{i}} c_{r}^{\leq i}(x, d)$. Let $G=(N, \bar{X}, \pi)$ be an arbitrary congestion game with variable demands and let $(x, d),(y, \Delta) \in \bar{X}$ be two strategy profiles such that $(x, d)=\left(\left(x_{k}, d_{k}\right),\left(x_{-k}, d_{-k}\right)\right)$ and $(y, \Delta)=\left(\left(y_{k}, \Delta\right),\left(x_{-k}, d_{-k}\right)\right)$ for some $x_{k}, y_{k} \in X_{k}, d_{k}, \Delta_{k} \in \mathbb{R}_{>0}, x_{-k} \in X_{-k}$ and $d_{-k} \in \mathbb{R}_{>0}^{n-1}$. We notice that $P_{i}(y, \Delta)=P_{i}(x, d)$ for all $i<k$. Now consider a player $i>k$. When computing $P_{i}(y, \Delta)-P_{i}(x, d)$, we observe that all costs corresponding to resources not contained in $x_{k} \cup y_{k}$ cancel out. For each resource $r \in\left(x_{i} \cap x_{k}\right) \backslash y_{k}$, we have $-c_{r}^{\leq i}(y, \Delta)+c_{r}^{\leq i}(x, d)=a_{r} d_{k} d_{i}$. Analogously, for each resource $r \in\left(x_{i} \cap y_{k}\right) \backslash x_{k}$, it holds that $-c_{r}^{\leq i}(y, \Delta)+c_{r}^{\leq i}(x, d)=-a_{r} \Delta_{k} d_{i}$. For each resource $r \in x_{i} \cap x_{k} \cap y_{k}$, we have $-c_{r}^{\leq i}(y, \Delta)+c_{r}^{\leq i}(x, d)=$ $a_{r}\left(d_{k}-\Delta_{k}\right) d_{i}$. Hence, for all players $i>k$, we have

$$
P_{i}(y, \Delta)-P_{i}(x, d)=-\sum_{r \in x_{i} \cap y_{k}} a_{r} \Delta_{k} d_{i}+\sum_{r \in x_{i} \cap x_{k}} a_{r} d_{k} d_{i}
$$


Moreover, we can calculate straightforwardly that

$$
\begin{aligned}
P_{k}(y, \Delta)-P_{k}(x, d)= & U_{k}\left(\Delta_{k}\right)-U_{k}\left(d_{k}\right)-\Delta_{k} \sum_{r \in y_{k}} c_{r}\left(\sum_{j \in\{1, \ldots, k-1\}: r \in y_{j}} d_{j}+\Delta_{k}\right) \\
& +d_{k} \sum_{r \in x_{k}} c_{r}\left(\sum_{j \in\{1, \ldots, k-1\}: r \in x_{j}} d_{j}+d_{k}\right)-\Delta_{k} \sum_{r \in y_{k}} b_{r}+d_{k} \sum_{r \in x_{k}} b_{r} .
\end{aligned}
$$

We thus obtain

$$
\begin{aligned}
P(y, \Delta)-P(x, d) & \\
= & \sum_{i \in N} P_{i}(y, \Delta)-\sum_{i \in N} P_{i}(x, d) \\
= & U_{k}\left(\Delta_{k}\right)-U_{k}\left(d_{k}\right)-\Delta_{k} \sum_{r \in y_{k}} a_{r}\left(\sum_{j \in\{1, \ldots, k-1\}: f \in x_{j}} d_{j}+\Delta_{k}\right)+d_{k} \sum_{r \in x_{k}} a_{r}\left(\sum_{j \in\{1, \ldots, k-1\}: r \in y_{j}} d_{j}+d_{k}\right) \\
& -\Delta_{k} \sum_{r \in y_{k}} b_{r}+d_{k} \sum_{r \in x_{k}} b_{r}+\sum_{i=k+1}^{n}\left(\sum_{r \in y_{i} \cap y_{k}} a_{r} \Delta_{k} d_{i}+\sum_{r \in x_{i} \cap x_{k}} a_{r} d_{k} d_{i}\right) \\
= & \sum_{r \in y_{k}} a_{r}\left(\sum_{j \in N \backslash\{k\}: r \in x_{j}} d_{j}+\Delta_{k}\right) \Delta_{k}-\sum_{r \in x_{k}} a_{r}\left(\sum_{j \in N \backslash\{k\}: r \in x_{j}} d_{j}+d_{k}\right) d_{k}-\Delta_{k} \sum_{f \in y_{k}} b_{r}+d_{k} \sum_{r \in x_{k}} b_{r} \\
= & \pi_{k}(y, \Delta)-\pi_{k}(y, d) .
\end{aligned}
$$

Thus, $P$ is an exact potential of $G$.

Since player $i$ 's marginal utility $U_{i}^{\prime}(d)$ is concave, there is $D_{i} \in \mathbb{R}_{>0}$ such that $U_{i}^{\prime}(d)<2 a_{c} d+b$ for all $c \in C$ and $d>D_{i}$. By Lemma 3.1 the demands $d_{i}>D_{i}$ are not payoff maximizing and thus strictly dominated. Hence, we can effectively restrict the set of demands of player $i$ to $\left[0, D_{i}\right]$. Because the potential function $P$ is continuous, $P$ attains its maximum on $X_{i \in N}\left(X_{i} \times\left[0, D_{i}\right]\right)$ and we derive that every congestion game with variable demands and affine costs admits a PNE.

To show the $\alpha$-FIP, let $\bar{x}^{0} \in \bar{X}$ be arbitrary and let $\gamma=\left(\bar{x}^{0}, \bar{x}^{1}, \ldots\right)$ be an $\alpha$-improvement path starting in $\bar{x}^{0}$. For the maximum of $P$ we have $m=\max _{\left.\bar{x} \in X_{i \in N}\left(X_{i},\left[0, D_{i}\right]\right)\right]} P(\bar{x})=\max _{\left.\bar{x} \in X_{i \in N}\left(X_{i},\left[0, \mathbb{R}_{\geq 0}\right]\right)\right]} P(\bar{x})$. Since $P$ is an exact potential we have $P\left(\bar{x}^{k+1}\right)-P\left(\bar{x}^{k}\right)>\alpha$. If $\gamma$ is infinite, we obtain that $P\left(\bar{x}^{k}\right)>m$ for all $k>\left(m-P\left(\bar{x}^{0}\right)\right) / \alpha$, which is a contradiction.

Homogeneously Exponential Cost Functions In the following lemma, we will prove that for every improvement step at least one of the following actions also yields an improvement: (i) adapting the configuration only while leaving the demand constant, (ii) adapting the demand only while leaving the used resources constant. Formally, we call a subset $I^{\prime} \subseteq I$ of improving moves essential if $\left\{\bar{y}:(\bar{x}, \bar{y}) \in I^{\prime}\right\}=\emptyset$ implies $\{\bar{y}:(\bar{x}, \bar{y}) \in I\}=\emptyset$ for all $\bar{x} \in \bar{X}$. Such subsets exist since the set of improving moves $I$ itself is essential.

A function $P: \bar{X} \rightarrow \mathbb{R}$ is called a local essential potential of $G$ if $\bar{x} \in \operatorname{argmax}_{\bar{y} \in \bar{X}} P(\bar{y})$ implies $\{\bar{y}:(\bar{x}, \bar{y}) \in$ $\left.I^{\prime}\right\}=\emptyset$. Clearly, $\bar{x}$ is a PNE.

Lemma 4.2. Let $G$ be congestion game with variable demands and homogeneously exponential costs. Then, $I^{\prime}=\left\{\left((x, d),\left(y_{i}, x_{-i}, d\right)\right) \in I\right\} \cup\left\{\left((x, d),\left(x, \Delta_{i}, d_{-i}\right)\right) \in I\right\}$ is an essential subset of improving moves.

Proof. For contradiction, let $i \in N$ and $\left(y_{i}, \Delta_{i}\right) \in \bar{X}_{i}$ be such that $\left((x, d),\left(y_{i}, x_{-i}, \Delta_{i}, d_{-i}\right) \in I\right.$ but both 
$\left((x, d),\left(y_{i}, x_{-i}, d\right)\right) \notin I^{\prime}$ and $\left((x, d),\left(x, \Delta_{i}, d_{-i}\right)\right) \notin I^{\prime}$. Then, we obtain

$$
\begin{aligned}
& \pi_{i}\left(\left(x_{i}, \Delta_{i}\right), \bar{x}_{-i}\right)-\pi_{i}\left(\left(x_{i}, d_{i}\right), \bar{x}_{-i}\right)=U_{i}\left(\Delta_{i}\right)-U_{i}\left(d_{i}\right)-\Delta_{i} e^{\phi \Delta_{i}} \sum_{r \in x_{i}} a_{r} e^{\phi \ell_{r}\left(\bar{x}_{-i}\right)}+d_{i} e^{\phi d_{i}} \sum_{r \in x_{i}} a_{r} e^{\phi \ell_{r}\left(\bar{x}_{-i}\right)} \leq 0, \\
& \pi_{i}\left(\left(y_{i}, d_{i}\right), \bar{x}_{-i}\right)-\pi_{i}\left(\left(x_{i}, d_{i}\right), \bar{x}_{-i}\right)=\quad-d_{i} e^{\phi d_{i}} \sum_{r \in y_{i}} a_{r} e^{\phi \ell_{r}\left(\bar{x}_{-i}\right)}+d_{i} e^{\phi d_{i}} \sum_{r \in x_{i}} a_{r} e^{\phi \ell_{r}\left(\bar{x}_{-i}\right)} \leq 0, \\
& \pi_{i}\left(\left(y_{i}, \Delta_{i}\right), \bar{x}_{-i}\right)-\pi_{i}\left(\left(x_{i}, d_{i}\right), \bar{x}_{-i}\right)=U_{i}\left(\Delta_{i}\right)-U_{i}\left(d_{i}\right)-\Delta_{i} e^{\phi \Delta_{i}} \sum_{r \in y_{i}} a_{r} e^{\phi \ell_{r}\left(\bar{x}_{-i}\right)}+d_{i} e^{\phi d_{i}} \sum_{r \in x_{i}} a_{r} e^{\phi \ell_{r}\left(\bar{x}_{-i}\right)}>0,
\end{aligned}
$$

where the latter inequality expresses the fact that $\left((x, d),\left(y_{i}, x_{-i}, \Delta_{i}, d_{-i}\right)\right) \in I(i)$. Subtracting (8) from (10) we obtain $-\Delta_{i} e^{\phi \Delta_{i}}\left(\sum_{r \in y_{i}} a_{r} e^{\phi \ell_{r}\left(\bar{x}_{-i}\right)}-\sum_{r \in x_{i}} a_{r} e^{\phi \ell_{r}\left(\bar{x}_{-i}\right)}\right)>0$, which is a contradiction to (9).

We will use the above Lemma to prove that every congestion game with variable demands and homogeneously exponential costs admits a local essential potential and thus a PNE.

Proposition 4.3. Let $C$ be a set of homogeneously exponential cost functions. Then, $C$ is consistent.

Proof. Let $G$ be a game with homogeneously exponential cost functions. Consider the function $\Phi(x, d)=$ $\sum_{i \in N} \int_{0}^{d_{i}} \frac{U_{i}^{\prime}(s)}{\phi s+1} d s-\sum_{r \in R} c_{r}(\ell(x, d))$. With the same arguments as in the proof of Proposition 4.1 we can find $D_{i} \in \mathbb{R}_{>0}$ such that $d_{i}^{*}<D_{i}$ holds for all $i \in N$ in every equilibrium $\left(x^{*}, d^{*}\right)$ of $G$. We claim that $\bar{x}^{*}=\left(x^{*}, d^{*}\right) \in \arg \max _{(x, d) \in \mathrm{X}_{i \in N}\left(x_{i},\left[0, D_{i}\right]\right)} P(x, d)$ is a PNE. Referring to Lemma 4.2 it is sufficient to show that there is no improving move from $\left(x^{*}, d^{*}\right)$ in which exclusively either the demand or the configuration of a single player is adapted.

Because $\left(x^{*}, d^{*}\right)$ maximizes $\Phi$, the optimality conditions give $\partial \Phi\left(x^{*}, d^{*}\right) / \partial d_{i}^{*}=0$, if $d_{i}^{*}>0$ and $\partial \Phi\left(x^{*}, d^{*}\right) / \partial d_{i}^{*} \leq 0$, if $d_{i}^{*}=0$. We thus obtain the equations $U_{i}^{\prime}\left(d_{i}^{*}\right)=\left(\phi d_{i}^{*}+1\right) \sum_{r \in x_{i}} a_{r} e^{\ell_{r}\left(x^{*}, d^{*}\right)}=$ $\partial d_{i}^{*} \sum_{r \in x_{i}} a_{r} e^{\phi \ell\left(x^{*}, d^{*}\right)} / \partial d_{i}^{*}$, if $d_{i}^{*}>0$, and $U_{i}^{\prime}\left(d_{i}^{*}\right) \leq\left(\phi d_{i}^{*}+1\right) \sum_{r \in x_{i}} a_{r} e^{\ell_{r}\left(x^{*}, d^{*}\right)}=\partial d_{i}^{*} \sum_{r \in x_{i}} a_{r} e^{\phi \ell}\left(x^{*}, d^{*}\right) / \partial d_{i}^{*}$, if $d_{i}^{*}=0$. Hence, the demand $d_{i}^{*}$ is optimal for player $i$ for the configuration profile $x^{*}$ implying that there is no improving move in which a player solely changes her demand.

Suppose that there is a player $k$ that changes profitably her configuration from $x_{k}^{*}$ to some $y_{k}$. Then,

$$
\begin{aligned}
\Phi\left(\left(y_{k}, d_{k}^{*}\right), \bar{x}_{-k}^{*}\right)-\Phi\left(\left(x_{k}^{*}, d_{k}^{*}\right), \bar{x}_{-k}^{*}\right) & =\left(\frac{1}{e^{\phi d_{k}^{*}}}-1\right)\left(\sum_{r \in y_{k}} a_{r} e^{\phi \ell\left(\bar{x}^{*}\right)}\right)+\left(1-\frac{1}{e^{\phi d_{k}^{*}}}\right)\left(\sum_{r \in x_{k}^{*}} a_{r} e^{\phi \ell\left(\bar{x}^{*}\right)}\right) \\
& =\left(\frac{1}{d_{k}^{*}}-\frac{1}{d_{k}^{*} e^{\phi d_{k}^{*}}}\right)\left(-d_{k}^{*} \sum_{r \in y_{k}} a_{r} e^{\phi \ell\left(\bar{x}^{*}\right)}+d_{k}^{*} \sum_{r \in x_{k}^{*}} a_{r} e^{\phi \ell\left(\bar{x}^{*}\right)}\right) \\
& =\left(\frac{1}{d_{k}^{*}}-\frac{1}{d_{k}^{*} e^{\phi d_{k}^{*}}}\right)\left(\pi_{k}\left(\left(y_{k}, d_{k}\right), \bar{x}_{-k}\right)-\pi_{k}\left(\left(x_{k}^{*}, d_{k}\right), \bar{x}_{-k}\right)\right)>0,
\end{aligned}
$$

which is a contradiction to the fact that $\left(x^{*}, d^{*}\right)$ maximizes $\Phi$. We derive that $\left(x^{*}, d^{*}\right)$ is a PNE.

We are now ready to state the main result of this paper.

Theorem 4.4. A set $C \subseteq C^{2}\left(\mathbb{R}_{\geq 0}\right)$ of strictly increasing cost functions is consistent if and only if one of the following cases holds: ( $i) C$ contains only affine functions $c(\ell)=a \ell+b, a>0, b \geq 0$; (ii) $C$ contains only homogeneously exponential functions $c(\ell)=a e^{\phi \ell}, a, \phi>0$, where $\phi$ is a universal constant of all functions in $C$.

Proof. Corollary 3.4 and Proposition 3.9 imply the "only if" part. Proposition 4.1 and Proposition 4.3 prove the "if" part.

The characterization of the set of FIP consistent cost functions follows from Proposition 3.7 
Theorem 4.5. A set $C \subseteq C^{2}\left(\mathbb{R}_{\geq 0}\right)$ of cost functions is FIP consistent if and only if $C$ contains only affine functions $c(\ell)=a \ell+b$ with $a>0, b \geq 0$.

Our characterizations also hold for network congestion games thus resolving the question raised in. [23].

\section{Pure Nash Equilibria in the Uniform Cost Model}

In the last section, we assumed that the cost function of a resource defines a per-unit-price for every player on that resource. Thus, the actual cost for a player is the product of the per-unit-price and the demand of that player. In this section, we will assume that the cost functions on the resources define uniform costs (or per-player costs), that is, every player pays the same costs regardless of her demand.

Let $\mathcal{M}=\left(N, R, X,\left(c_{r}\right)_{r \in R}\right)$ be a congestion model and let $\left(U_{i}\right)_{i \in N}$ be a collection of utility functions. We define the corresponding uniform congestion game with variable demands as the strategic game $G(\mathcal{M})=$ $(N, \bar{X}, \pi)$, where $\bar{X}=\left(X, \mathbb{R}_{\geq 0}\right), \pi=\chi_{i \in N} \pi_{i}$ and $\pi_{i}(x, d)=U_{i}\left(d_{i}\right)-\sum_{r \in x_{i}} c_{r}\left(\ell_{r}(x, d)\right)$, and $\ell_{r}((x, d))=$ $\sum_{j \in N: r \in x_{j}} d_{j}$.

Most surprisingly, in the uniform cost model, there is a congestion game with variable demands and linear cost functions without PNE, as illustrated in the following example.

Example 2 (Uniform cost game without PNE). Consider the congestion model $\mathcal{M}=\left(N, R, X,\left(c_{r}\right)_{r \in R}\right)$, where $N=\{1,2\}$ and $R=\{1, \ldots, 46\}$. Each resource has the cost function $c(\ell)=\ell$. We define $X=X_{i \in N} X_{i}$, where

$$
\begin{aligned}
& X_{1}=\left\{\left\{r_{1}, \ldots, r_{22}\right\},\left\{r_{23}, \ldots, r_{39}\right\}\right\} \\
& X_{2}=\left\{\left\{r_{23}, \ldots, r_{30}\right\} \cup\left\{r_{40}, \ldots, r_{46}\right\},\left\{r_{1}, \ldots, r_{4}\right\} \cup\left\{r_{31}, \ldots, r_{39}\right\}\right\} .
\end{aligned}
$$

For ease of exposition, we define $R_{1,2}=\left\{r_{1}, \ldots, r_{4}\right\}, R_{1,0}=\left\{r_{5}, \ldots, r_{22}\right\}, R_{2,1}=\left\{r_{23}, \ldots, r_{30}\right\}, R_{2,2}=$ $\left\{r_{31}, \ldots, r_{39}\right\}, R_{0,1}=\left\{r_{40}, \ldots, r_{46}\right\}$. The strategy space is depicted in Figure 4

For the utility functions $U_{1}$ and $U_{2}$ of player 1 and 2 , respectively we chose strictly concave, increasing and twice continuously differentiable functions satisfying

$$
\begin{array}{llrl}
U_{1}(0)=0, & U_{1}(2)=53, \\
U_{1}^{\prime}(1)=32, & U_{1}^{\prime}(2)=17, \\
U_{2}(0)=0, & U_{2}(2)=46, \\
U_{2}(1)=32, & U_{2}^{\prime}(2)=13 .
\end{array}
$$

Clearly, such functions always exists, for instance one can choose polynomials of degree seven to fit the equations above. These functions are shown in Figure 5

Using that the cost functions are equal to the identity, the equilibrium conditions for the Nash equilibrium $\left(x^{*}, d^{*}\right)$ imply $U_{i}^{\prime}\left(d_{i}^{*}\right)=\left|x_{i}\right|$ for $d_{i}^{*}>0$. Said differently, in any PNE in which player i plays configuration $x_{i}^{*}$ and has positive demand $d_{i}^{*}$, the equation $d_{i}^{*}=U_{i}^{\prime-1}\left(\left|x_{i}^{*}\right|\right)$ holds. Note that since the utility functions are strictly concave, the derivatives $U_{1}^{\prime}$ and $U_{2}^{\prime}$ are convertible and thus their inverse functions $U_{1}^{\prime-1}$ and $U_{2}^{\prime-1}$ are well-defined. Now, remark that the utility functions $U_{i}$ of player $i$ is chosen such that in equilibrium $d_{i}^{*} \in\{0,1,2\}$, since

$$
\begin{array}{ll}
U_{1}^{\prime-1}\left(\left|R_{1,2} \cup R_{1,0}\right|\right)=1, & U_{1}^{\prime-1}\left(\left|R_{2,1} \cup R_{2,2}\right|\right)=2, \\
U_{2}^{\prime-1}\left(\left|R_{2,1} \cup R_{0,1}\right|\right)=1, & U_{2}^{\prime-1}\left(\left|R_{1,2} \cup R_{2,2}\right|\right)=2 .
\end{array}
$$




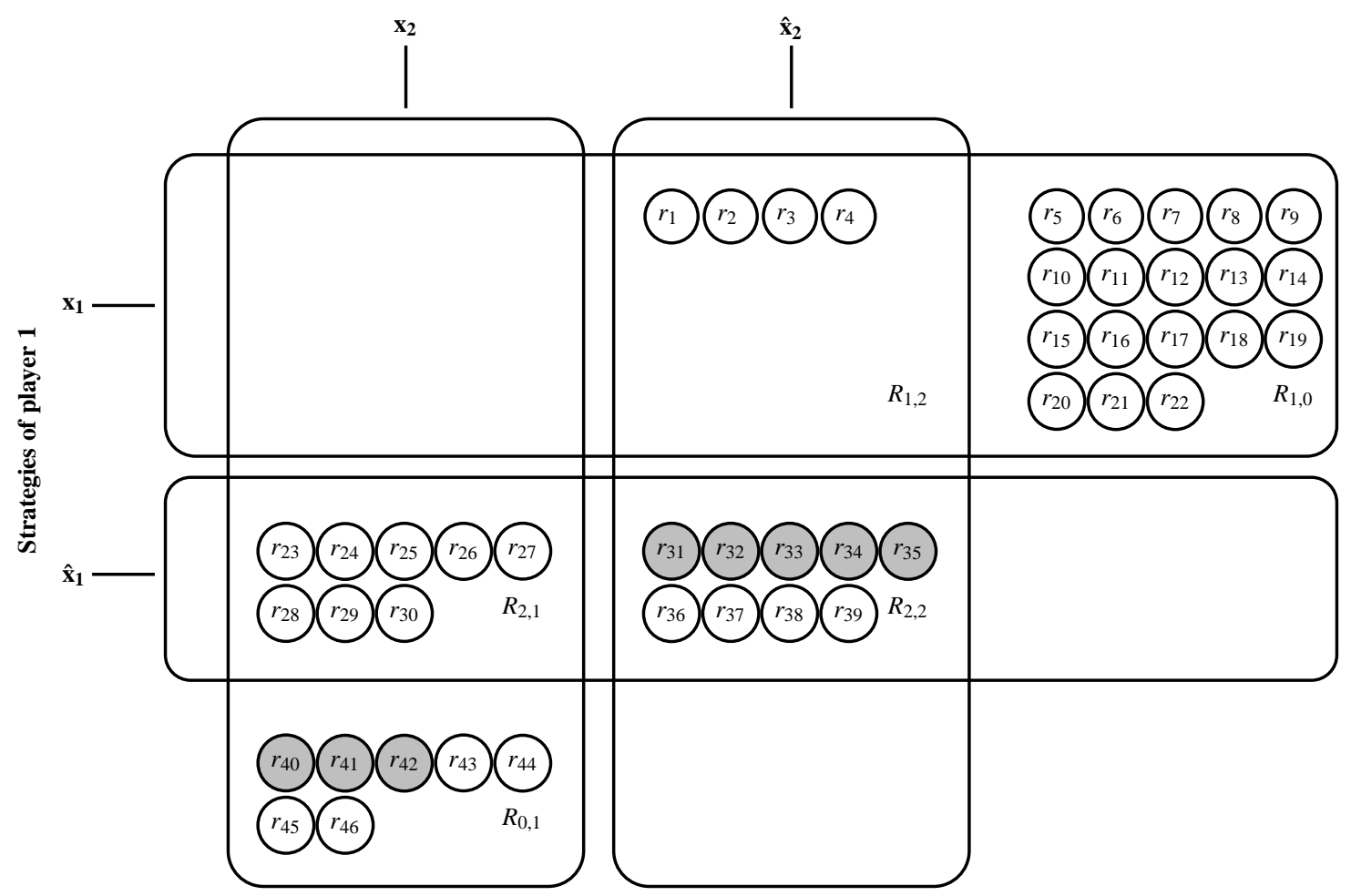

Figure 4: Strategies of the uniform cost congestion game with variable demands with identity cost and no PNE considered in Example 2. The gray shaded resources are important in the construction of Proposition 5.2

Thus, for any equilibrium $\left(x^{*}, d^{*}\right)$ we get

$$
\begin{gathered}
\left(x_{1}^{*}, d_{1}^{*}\right) \in\left\{\left(R_{1,2} \cup R_{1,0}, 1\right),\left(R_{2,1} \cup R_{2,2}, 2\right),\left(R_{1,2} \cup R_{1,0}, 0\right),\left(R_{2,1} \cup R_{2,2}, 0\right)\right\}, \\
\left(x_{2}^{*}, d_{2}^{*}\right) \in\left\{\left(R_{2,1} \cup R_{0,1}, 1\right),\left(R_{1,2} \cup R_{2,2}, 2\right),\right. \\
\left.\left(R_{2,1} \cup R_{0,1}, 0\right),\left(R_{1,2} \cup R_{2,2}, 0\right)\right\} .
\end{gathered}
$$

Remark that the latter two strategies of players 1 and 2 with zero demand are indistinguishable in the sense that they always give the same payoff to every player. First, we will show that there is no PNE in which both players chose a strictly positive demand. We calculate that

$$
\begin{aligned}
& \pi_{1}\left(\left(R_{2,1} \cup R_{2,2}, 2\right),\left(R_{2,1} \cup R_{0,1}, 1\right)\right)-\pi_{1}\left(\left(R_{1,2} \cup R_{1,0}, 1\right),\left(R_{2,1} \cup R_{0,1}, 1\right)\right) \\
& \quad=U_{1}(2)-3 \cdot\left|R_{2,1}\right|-2 \cdot\left|R_{2,2}\right|-\left(U_{1}(1)-1 \cdot\left|R_{1,2}\right|-1 \cdot\left|R_{1,0}\right|\right) \\
& \quad=(53-3 \cdot 8-2 \cdot 9)-(32-1 \cdot 4-1 \cdot 18)=11-10=1,
\end{aligned}
$$

and similarly

$$
\begin{aligned}
& \pi_{2}\left(\left(R_{2,1} \cup R_{2,2}, 2\right),\left(R_{1,2} \cup R_{2,2}, 2\right)\right)-\pi_{2}\left(\left(R_{2,1} \cup R_{2,2}, 2\right),\left(R_{2,1} \cup R_{0,1}, 1\right)\right)=2-1=1, \\
& \pi_{1}\left(\left(R_{1,2} \cup R_{1,0}, 1\right),\left(R_{1,2} \cup R_{2,2}, 2\right)\right)-\pi_{1}\left(\left(R_{2,1} \cup R_{2,2}, 2\right),\left(R_{1,2} \cup R_{2,2}, 2\right)\right)=2-1=1, \\
& \pi_{2}\left(\left(R_{1,2} \cup R_{1,0}, 1\right),\left(R_{2,1} \cup R_{0,1}, 1\right)\right)-\pi_{2}\left(\left(R_{1,2} \cup R_{1,0}, 1\right),\left(R_{1,2} \cup R_{2,2}, 2\right)\right)=17-16=1,
\end{aligned}
$$

establishing that neither of these 4 strategy profiles constitutes a PNE. Note that each of these strategy profiles guarantees a strictly positive payoff to each player while choosing $d_{i}^{*}=0$ gives a payoff equal to zero. Hence, both players have an incentive to choose a positive demand which together with the observation that $\gamma$ is an improvement cycle establishes that this game does not admit a PNE.

This is in stark contrast to Proposition 4.1 establishing that every congestion game with variable demand in the proportional cost model possesses a PNE. We will shed light on this dichotomy by obtaining a complete characterization. 


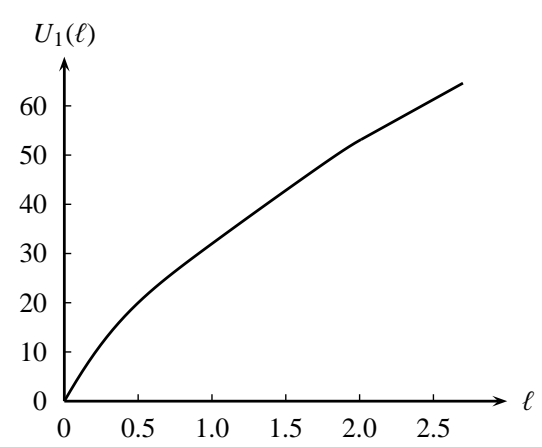

(a) Utility function of player 1 .

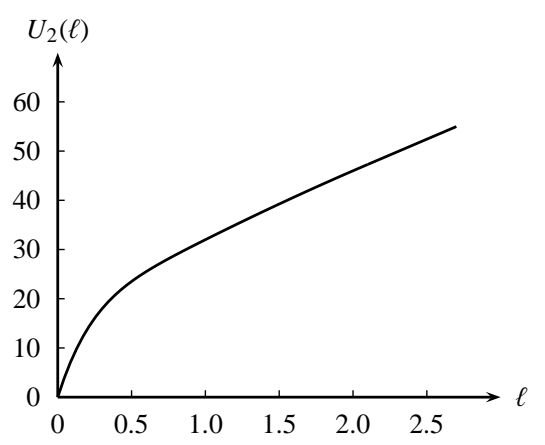

(b) Utility function of player 2.

Figure 5: Utility functions $U_{1}$ and $U_{2}$ of players 1 and 2, respectively, considered in Example 2

Necessary Conditions. As in the proportional cost model, we can restrict our search space for consistent cost functions to affine and exponential functions. With a slight modification in the proof of Proposition 3.3 one can establish that every functions that is not consistent w.r.t. weighted congestion games is also non-consistent w.r.t. uniform congestion games with variable demands. Like this, we get the following immediate corollary.

Corollary 5.1. If $C \subseteq C^{2}\left(\mathbb{R}_{\geq 0}\right)$ is consistent w.r.t. uniform congestion games with variable demands then one of the following cases holds: (i) $C$ contains only affine functions; (ii) $C$ contains only exponential functions.

While the upper result seemingly establishes a structural similarity between the games with proportional costs, it turns out that they behave completely different. As already mentioned, uniform congestion games variable demands and affine costs need not possess a PNE. This holds even for two player games in which the cost function $c_{r}$ on all resources $r \in R$ equals the identity as illustrated in Example 2. Using this example as a blueprint, one can show the following more general result.

Proposition 5.2. Any affine function is not consistent w.r.t. uniform congestion games with variable demands.

Proof. In Example 2, we construct a uniform congestion game $G$ with variable demands and costs equal to $c(\ell)=\ell$ that does not admit a PNE. Now, consider the game $G^{a}$ with utility functions $U_{1}^{a}$ and $U_{2}^{a}$, where $U_{1}^{a}=a U_{1}$ and $U_{2}^{a}=a U_{2}$ for some $a>0$. Furthermore, we set the cost on all resources equal to $c(\ell)=a \ell$. Clearly, the utility of every player in $G$ is equal to the scaled utility of that player in $G^{a}$. Thus, neither $G$ nor $G^{a}$ admit a PNE.

In order to construct a game $G^{a, b}$ with costs equal to $c(\ell)=a \ell+b$ we introduce a third player with utility equal to $U_{3}^{a, b}=g^{\sigma}((0,0),(b, M))$ and a single configuration $X_{3}=\{\{31, \ldots, 35\} \cup\{40,41,42\}\}$. For illustration, these resources are shaded gray in Fig 4 With similar arguments as before, we get that for sufficiently large $M$ and sufficiently small $\sigma$, player 3 will choose her demand $d_{3}^{*}$ in any equilibrium (if such exists) such that $d_{3}^{*} \in(b-\sigma, b+\sigma)$. We define the utility functions $U_{1}^{a, b}$ and $U_{2}^{a, b}$ in the game $G^{a, b}$ by $U_{1}^{a, b}=U_{1}^{a}+22 b$ and $U_{2}^{a, b}=U_{2}+18 b$. It is easy to check that the difference of the payoffs of the games $G^{a, b}$ and $G^{a}$, respectively, goes to 0 as $\sigma \rightarrow 0$. Using that the payoff functions of the game $G^{a, b}$ are continuous with respect to $\sigma$, we derive that the game $G^{a, b}$ does not possess a PNE.

In order to show a negative result for inhomogenously exponential functions, we will follow the same line of argumentation as for the proportional cost model. To this end, we first introduce the notion of uniform 
congestion games with resource dependent demands. Let $\mathcal{M}=\left(N, F, X,\left(c_{r}\right)_{r \in R}\right)$ be a congestion model and let $\left(d_{i, r}\right)_{i \in N, r \in R}$ be a matrix of facility-dependent demands. The corresponding uniform congestion game with resource dependent demands is the strategic game $G(\mathcal{M})=(N, X, \pi)$, where $\pi$ is defined as $\pi=\chi_{i \in N} \pi_{i}$, $\pi_{i}(x)=\sum_{r \in x_{i}} c_{r}\left(\ell_{r}(x)\right)$ and $\ell_{r}(x)=\sum_{j \in N: r \in x_{j}} d_{j, r}$.

Proposition 5.3. Any inhomogenously exponential function is not consistent w.r.t. uniform congestion games with resource dependent demands.

Proof. Let $c(\ell)=a e^{\phi \ell}+b$ with $a, \phi \in \mathbb{R}_{>0}$ and $b \geq-a$ and consider the congestion model $\mathcal{M}=$ $\left(N, R, X,\left(c_{r}\right)_{r \in R}\right)$ with 5 players and 7 resources $R=\left\{r_{1}, r_{2}, r_{3}, r_{4}, r_{5}, r_{6}, r_{7}\right\}$. Only the first two players $i \in\{1,2\}$ have two strategies and will be called non-trivial players in the sequel of this proof. Their strategy spaces are given by

$$
\begin{aligned}
& X_{1}=\left\{x_{1}, \hat{x}_{1}\right\}, \quad \text { where } \quad x_{1}=\left\{r_{1}, r_{2}\right\}, \quad \hat{x}_{1}=\left\{r_{3}, r_{4}\right\}, \\
& X_{2}=\left\{x_{2}, \hat{x}_{2}\right\}, \quad \text { where } \quad x_{2}=\left\{r_{1}, r_{2}, r_{3}\right\}, \quad \hat{x}_{2}=\left\{r_{5}, r_{6}, r_{7}\right\} \text {. }
\end{aligned}
$$

The players 3, 4, and 5 have only one strategy and serve only to increase the costs on some of the resources. They will be called trivial players. Their strategies are given by $X_{3}=\left\{x_{3}\right\}=\left\{\left\{r_{1}, r_{2}\right\}\right\}, X_{4}=\left\{x_{4}\right\}=\left\{r_{4}\right\}$, $X_{5}=\left\{x_{5}\right\}=\left\{\left\{r_{5}, r_{6}, r_{7}\right\}\right\}$. Observe that the strategies of the two non-trivial players are disjoint. For the nontrivial players $i \in\{1,2\}$ and the parameters $p, q \in \mathbb{N}_{>0}$, we set $d_{i, r}=2 \frac{\ln 2}{\phi}$ for all $r \in x_{i}$ and $d_{i, r}=\left(2+\frac{p}{q}\right) \frac{\ln 2}{\phi}$ for all $r \in \hat{x}_{i}$.

We set the demand of the non-trivial players 3,4 , and 5 equal to $d_{3, r}=\ln \left(a_{3}\right) / \phi, d_{4, r}=\ln \left(a_{4}\right) / \phi$, and $d_{5, r}=\ln \left(a_{5}\right) / \phi$ for all $r \in R$, where $a_{3}, a_{4}, a_{5}>1$ will be chosen such that

$$
\gamma=\left(\left(x_{1}, x_{2}, x_{3}, x_{4}, x_{5}\right),\left(\hat{x}_{1}, x_{2}, x_{3}, x_{4}, x_{5}\right),\left(\hat{x}_{1}, \hat{x}_{2}, x_{3}, x_{4}, x_{5}\right),\left(x_{1}, \hat{x}_{2}, x_{3}, x_{4}, x_{5}\right),\left(x_{1}, x_{2}, x_{3}, x_{4}, x_{5}\right)\right)
$$

is an improvement cycle. Calculating the costs of the respective deviating non-trivial player in $\gamma$, we obtain the following necessary and sufficient conditions

$$
\begin{aligned}
2^{5} a_{3} & >2^{4+p / q} a_{4}+2^{2+p / q} \\
2^{3} a_{3}+2^{4+p / q} a_{4} & >3 \cdot 2^{2+p / q} a_{5} \\
2^{2+p / q} a_{4}+2^{2+p / q} & >2^{3} a_{3} \\
3 \cdot 2^{2+p / q} a_{5} & >2^{5} a_{3}+2^{2} a_{4} .
\end{aligned}
$$

Combining (U2) and (U4) into one inequality, we obtain

$$
\begin{gathered}
2 a_{3}+2^{2+p / q} a_{4}>3 \cdot 2^{p / q} a_{5}>2^{3} a_{3}+a_{4} \\
\Rightarrow a_{3}<\left(\frac{2}{3} 2^{p / q}-\frac{1}{6}\right) a_{4} .
\end{gathered}
$$

Inequalities (U1) and (U3) give rise to

$$
\begin{aligned}
\frac{1}{2} 2^{p / q} a_{4}+\frac{1}{8} 2^{p / q} & <a_{3} \\
a_{3} & <\frac{1}{2} 2^{p / q} a_{4}+\frac{1}{2} 2^{p / q}
\end{aligned}
$$

respectively. It is left to show that the inequalities (U1'), (U2'), and (U3') can be satisfied simultaneously. Let us choose $a_{4}>\max \left\{2^{1+p / q}, \frac{3}{2}\left(1+\frac{1}{2^{p / q}-1}\right)\right\}$ and $a_{3}=\frac{1}{2} 2^{p / q} a_{4}+\frac{1}{4} 2^{p / q}$. Then, (U1') and (U3') are satisfied trivially. As for (U2), note that

$$
\frac{2}{3} 2^{p / q} a_{4}-\frac{1}{6} a_{4}-a_{3}=\frac{2}{3} 2^{p / q} a_{4}-\frac{1}{6} a_{4}-\frac{1}{2} 2^{p / q} a_{4}-\frac{1}{4} 2^{p / q}=\frac{1}{6} a_{4}\left(2^{p / q}-1\right)-\frac{1}{4} 2^{p / q}>0
$$


by the choice of $a_{4}$. Finally, we set $a_{5}=\frac{1}{3 \cdot 2^{p / q}}\left(\left(2^{1+p / q}-1\right) a_{4}-3 a_{3}\right)$. It is left to show, that $a_{3}, a_{4}$, and $a_{5}$ are not smaller than 1 . Since $a_{4}>2^{1+p / q}$ by definition, $a_{3}>2^{p / q}>1$. As for $a_{5}$, inequality (U4) gives rise to $2^{p / q} a_{5}>2 a_{3}$ implying $a_{5}>1$.

Thus, we have found $a_{3}, a_{4}, a_{5}>1$ such that $\gamma$ is an improvement cycle, establishing that the corresponding game does not admit a PNE.

Using the same idea as in the proof of Proposition 3.9 the non-consistency of inhomogenously exponential functions carries over to uniform congestion games with variable demands.

Proposition 5.4. Any inhomogenously exponential function is not consistent w.r.t. uniform congestion games with variable demands.

Sufficient Conditions. For uniform congestion games with variable demands and homogenous exponential costs, we establish the existence of a PNE by deriving an essential potential function which generalize the potentials considered by Monderer and Shapley [21]. Recall that a subset $I^{\prime} \subseteq I$ of improving moves is essential if $\left\{\bar{y}:(\bar{x}, \bar{y}) \in I^{\prime}\right\}=\emptyset$ implies $\{\bar{y}:(\bar{x}, \bar{y}) \in I\}=\emptyset$ for all $\bar{x} \in \bar{X}$. This definition motivates the concept of essential generalized ordinal potentials. Let $G=(N, \bar{X}, \pi)$ be a strategic game. The function $P: \bar{X} \rightarrow \mathbb{R}$ is called an essential generalized ordinal potential of $G$ if for all strategy profiles $\bar{x} \in \bar{X}$ there is an essential subset of improving moves $I^{\prime}$ such that $(\bar{x}, \bar{y}) \in I^{\prime}$ implies $P(\bar{y})>P(\bar{x})$. Clearly, a maximizer of $P$ is a PNE.

Proposition 5.5. Let $G=(N, \bar{X}, \pi)$ be a uniform congestion game with variable demands and homogenous exponential costs. Then, $G$ admits an essential generalized ordinal potential and possesses a PNE.

Combining Corollary 5.1 and Propositions 5.2, 5.4, and 5.5, we state the main result of this section.

Theorem 5.6. A set $C \subseteq C^{2}\left(\mathbb{R}_{\geq 0}\right)$ of cost functions is consistent w.r.t. uniform congestion games with variable demands if and only if $C$ contains only homogeneously exponential functions. There is no set of FIP consistent functions.

\section{Conclusions and Open Problems}

We considered the fundamental problem of the existence of PNE and convergence properties of improvement dynamics in congestion games with variable demands. We obtained several characterizations of the cost structure with respect to the existence of PNE and the $\alpha$-FIP. Since our model is general enough to closely capture many elements of practical applications, we are confident that our results help understanding the behavior of myopic play in real systems. We conclude the paper by outlining several research directions that deserve further attention.

While the present work addressed the existence of PNE (and the $\alpha$-FIP) with respect to the cost structure (without constraining the strategy space and the utility functions), it is natural to ask for combinatorial properties of the strategy spaces (such as singletons strategies or strategies given by bases of a matroid) that ensure the existence of PNE for general cost functions. Alternatively, one can restrict the set of feasible utility functions (e.g., assume linear functions) and ask for the existence of PNE. Also the case of symmetry within the set of players (with respect to their utilities, their strategies or both) is open.

Another research direction is to investigate the price of anarchy (stability) in congestion games with variable demands. It would be interesting to characterize the price of anarchy for affine cost functions, because in this case there always exists a PNE.

The design and the analysis of improvement dynamics is fundamental and deserves further investigation. Because our model captures many elements of TCP/IP routing protocols (involving unsplittable routings and elastic demands), it is of practical importance to further investigate the stability and scalability of distributed improvement dynamics. 


\section{References}

[1] H. Ackermann, H. Röglin, and B. Vöcking. Pure Nash equilibria in player-specific and weighted congestion games. Theor. Comput. Sci., 410(17):1552-1563, 2009.

[2] E. Altman, T. Basar, T. Jimnez, and N. Shimkin. Competitive routing in networks with polynomial costs. IEEE Trans. Autom. Control, 47:92-96, 2002.

[3] E. Anshelevich, A. Dasgupta, J. Kleinberg, É. Tardos, T. Wexler, and T. Roughgarden. The price of stability for network design with fair cost allocation. SIAM J. Comput., 38(4):1602-1623, 2008.

[4] M. Beckmann, C. McGuire, and C. Winsten. Studies in the Economics and Transportation. Yale University Press, 1956.

[5] H. Chen and T. Roughgarden. Network design with weighted players. Theor. Comput. Syst., 45(2):302324, 2009.

[6] R. Cole, Y. Dodis, and T. Roughgarden. Bottleneck links, variable demand, and the tragedy of the commons. In Proc. 17th Annual ACM-SIAM Sympos. on Discrete Algorithms, pages 668-677, 2006.

[7] A. Cournot. Recherches Sur Les Principes Mathematiques De La Theorie De La Richesse. Hachette, Paris, 1838.

[8] D. Fotakis, S. Kontogiannis, and P. Spirakis. Selfish unsplittable flows. Theor. Comput. Sci., 348(23):226-239, 2005.

[9] M. Gairing, B. Monien, and K. Tiemann. Routing (un-) splittable flow in games with player-specific linear latency functions. In M. Bugliesi, B. Preneel, V. Sassone, and I. Wegener, editors, Proc. 33rd Internat. Colloquium on Automata, Languages and Programming, volume 4051 of LNCS, pages 501$512,2006$.

[10] M. Goemans, V. Mirrokni, and A. Vetta. Sink equilibria and convergence. In Proc. 46th Annual IEEE Sympos. Foundations Comput. Sci., pages 142-154, 2005.

[11] T. Harks and M. Klimm. On the existence of pure Nash equilibria in weighted congestion games. In S. Abramsky, C. Gavoille, C. Kirchner, F. Meyer auf der Heide, and P. Spirakis, editors, Proc. 37rd Internat. Colloquium on Automata, Languages and Programming, volume 6198 of LNCS, pages 79 89, 2010.

[12] T. Harks, M. Klimm, and R. Möhring. Characterizing the existence of potential functions in weighted congestion games. In M. Mavronicolas and V. Papadopoulou, editors, Proc. 2nd Internat. Sympos. Algorithmic Game Theory, volume 5814 of LNCS, pages 97 - 108, 2009.

[13] A. Haurie and P. Marcotte. On the relationship between Nash-Cournot and Wardrop equilibria. Networks, 15:295-308, 1985.

[14] S. Ieong, R. McGrew, E. Nudelman, Y. Shoham, and Q. Sun. Fast and compact: A simple class of congestion games. In Proc. 20th National Conf. Artificial Intelligence and the 17th Innovative Appl. Artificial Intelligence Conf., pages 489-494, 2005.

[15] R. Johari and J. Tsitsiklis. Efficiency loss in cournot games. Technical report, LIDS-P-2639, Laboratory for Information and Decision Systems, MIT, 2005. 
[16] R. Johari and J. Tsitsiklis. A scalable network resource allocation mechanism with bounded efficiency loss. IEEE J. Sel. Areas Commun., 24(5):992-999, 2006.

[17] F. Kelly, A. Maulloo, and D. Tan. Rate Control in Communication Networks: Shadow Prices, Proportional Fairness, and Stability. J. Oper. Res. Soc., 49:237-52, 1998.

[18] L. Libman and A. Orda. Atomic resource sharing in noncooperative networks. Telecommunication Systems, 17(4):385-409, 2001.

[19] S. Low and D. Lapsley. Optimization flow control i: Basic algorithm and convergence. IEEE/ACM Trans. Networking, 7:861-874, 1999.

[20] I. Milchtaich. The equilibrium existence problem in finite network congestion games. In M. Mavronicolas and S. Kontogiannis, editors, Proc. 2nd Internat. Workshop on Internet and Network Econom., volume 4286 of $L N C S$, pages 87 - 98, 2006.

[21] D. Monderer and L. Shapley. Potential games. Games Econom. Behav., 14(1):124 - 143, 1996.

[22] W. Novshek. On the existence of Cournot equilibrium. Rev. Econom. Stud., 52(1):85-98, 1985.

[23] A. Orda, R. Rom, and N. Shimkin. Competitive routing in multi-user communication networks. IEEE Transactions on Networking, 1:510-521, 1993.

[24] P. Panagopoulou and P. Spirakis. Algorithms for pure Nash equilibria in weighted congestion games. ACM J. Exp. Algorithmics, 11(2.7):1-19, 2006.

[25] J. Roberts and H. Sonnenschein. On the existence of Cournot equilibrium without concave profit functions. J. Econom. Theory, 22:112-117, 1976.

[26] J. Rosen. Existence and uniqueness of equilibrium points in concave n-player games. Econometrica, 33(3):520-534, 1965.

[27] R. Rosenthal. A class of games possessing pure-strategy Nash equilibria. Internat. J. Game Theory, 2(1):65-67, 1973.

[28] T. Roughgarden. Selfish Routing and the Price of Anarchy. The MIT Press, 2005.

[29] S. Shenker. Fundamental design issues for the future internet. IEEE J. Sel. Areas Commun., 13:11761188, 1995.

[30] M. Smith. The marginal cost taxation of a transportation network. Transp. Res. Part B: Methodological, 13(3):237-242, 1979.

[31] R. Srikant. The Mathematics of Internet Congestion Control. Birkhäuser Boston, 2003.

[32] J. Wardrop. Some theoretical aspects of road traffic research. Proc. of the Institution of Civil Engineers, 1(Part II):325-378, 1952. 